\title{
Contrast Agents for Magnetic Resonance Imaging Synthesized with Ring- Opening Metathesis Polymerization
}

\author{
Matthew J. Allen, Ronald T. Raines, and Laura L. Kiessling* \\ Contribution from the Departments of Chemistry and Biochemistry, University of \\ Wisconsin-Madison, Madison, WI 53706 \\ E-mail: kiessling@chem.wisc.edu
}

$\begin{array}{ll}\text { Page } & \text { Contents } \\ \text { S1 } & \text { Table of Contents } \\ \text { S2-S13 } & \text { Experimental Procedures } \\ \text { S13-S14 } & \text { Estimation of Rotational Correlation Time } \\ \text { S14 } & \text { References } \\ \text { S15-S42 } & { }^{1} \mathrm{H} \text { and }{ }^{13} \mathrm{C} \text { NMR spectra }\end{array}$




\section{Experimental Procedures}

Commercial chemicals were of reagent grade or better and used without further purification unless otherwise noted. Dichloromethane and diisopropylethylamine were distilled from calcium hydride; tetrahydrofuran (THF) was distilled from sodium/benzophenone, and methanol was distilled from magnesium.

Flash chromatography was performed using silica gel 60, 230-450 mesh (Sorbent Technologies). Analytical thin-layer chromatography (TLC) was carried out on EM Science TLC plates precoated with silica gel $60 \mathrm{~F}_{254}(250-\mu \mathrm{m}$ layer thickness). TLC visualization was accomplished using a UV lamp and/or charring with potassium permanganate stain $\left(3 \mathrm{~g} \mathrm{KMnO}_{4}\right.$, $20 \mathrm{~g} \mathrm{~K}_{2} \mathrm{CO}_{3}, 5 \mathrm{~mL} \mathrm{5 \%}(\mathrm{w} / \mathrm{v})$ aqueous $\mathrm{NaOH}, 300 \mathrm{~mL} \mathrm{H}_{2} \mathrm{O}$ ). MilliQ water and PD-10 columns (Amersham Biosciences) were used for polymer purification.

${ }^{1} \mathrm{H}$ NMR spectra were obtained using a Bruker AC-300 (300 MHz) or Varian UNITY$500(500 \mathrm{MHz})$ spectrometer, and ${ }^{13} \mathrm{C}$ NMR spectra were obtained using a Bruker AC-300 (75 $\mathrm{MHz})$ spectrometer. Chemical shifts are reported relative to residual solvent signals $\left(\mathrm{CDCl}_{3}:{ }^{1} \mathrm{H}\right.$ : $\delta 7.27,{ }^{13} \mathrm{C}: \delta 77.23 ; \mathrm{CD}_{3} \mathrm{OD}:{ }^{1} \mathrm{H}: \delta 3.31,{ }^{13} \mathrm{C}: \delta 49.15$; DMSO- $d_{6}:{ }^{1} \mathrm{H}: \delta 2.50,{ }^{13} \mathrm{C}: \delta 39.51 ; \mathrm{D}_{2} \mathrm{O}$ : ${ }^{1} \mathrm{H}: \delta 4.79,{ }^{13} \mathrm{C}: \delta 39.51-$ internal DMSO- $d_{6}$ standard). $1 \mathrm{H}$ NMR data are assumed to be first order with apparent doublets and triplets reported as $d$ and $t$, respectively. Multiplets are reported as $\mathrm{m}$, and resonances that appear broad are designated as bs. High-resolution electrospray ionization mass spectra (HRESI-MS) were obtained on a Micromass LCT. Liquid chromatography and mass spectrometry (LC-MS) analysis was performed on a Shimadzu LCMS containing a C18 column (Supelco Discovery, 2.1 X $150 \mathrm{~mm}$ ) equilibrated with $0.4 \%$ (v/v) formic acid.

Polydispersity index (PDI) values were obtained using a Beckman Coulter highperformance liquid chromatography system, two Polymer Laboratories PLgel $5 \mu \mathrm{m}$ MIXED-D $300 \times 7.5 \mathrm{~mm}$ columns in series, Polymer laboratories EasiCal Polystyrene Standards (PS-1), and Cirrus GPC offline GPC/SEC Software Version 1.2. Elemental analyses and Gd solution concentration determinations were performed at Desert Analytics Laboratory, Tucson, AZ.

The longitudinal water proton relaxation rate at $60 \mathrm{MHz}$ was measured by using a Bruker mq60 NMR Analyzer (Bruker Canada, Milton, Ont. Canada) operating at $1.5 \mathrm{~T}$, by means of the standard inversion-recovery technique (20 data points, 8 scans each). A typical $90^{\circ}$-pulse length was $6.16 \mu \mathrm{s}$, and the reproducibility of the $T_{1}$ data was $\pm 0.3 \%$. Temperature was maintained at $22{ }^{\circ} \mathrm{C}$ with a Haake $\mathrm{G}$ cooling circulator.

3-Hydroxy-6-methyl-2-oxo-1,2-dihydropyridine-4-carboxylic acid ethyl ester (4), ${ }^{1} 10$ methoxydec-9-en-2-one, ${ }^{2}$ and $\left(\mathrm{H}_{2} \mathrm{IMes}\right)(3-\mathrm{Br}-\mathrm{py})_{2}(\mathrm{Cl})_{2} \mathrm{Ru}=\mathrm{CHPh}^{3}$ were synthesized following previously described procedures.

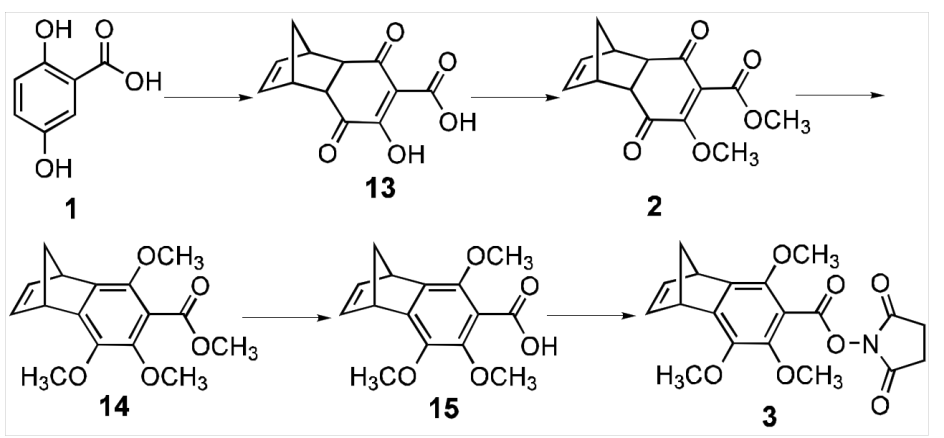




\section{7-Hydroxy-5,8-dioxo-1,4,4 $\alpha, 5,8,8 \alpha$-hexahydro-1,4-methanonaphthalene-6- carboxylic acid (13):}<smiles></smiles>

Modifications to a previously described procedure were used. ${ }^{4}$ A suspension of 2,5dihydroxybenzoic acid $(40.0 \mathrm{~g}, 0.263 \mathrm{~mol})$ and ammonium cerium(IV) sulfate $(315 \mathrm{~g}, 0.528 \mathrm{~mol})$ in carbon tetrachloride $(3 \mathrm{~L}$ ) was mechanically stirred rapidly in the dark for $45 \mathrm{~min}$. The suspension was filtered, and freshly cracked cyclopentadiene $(50 \mathrm{~mL}, 0.61 \mathrm{mmol})$ was added to the filtrate, and the mixture was allowed to stir. The solution color immediately changed from dark to light yellow. After $10 \mathrm{~min}$, the solvent was removed under reduced pressure. The resulting orange solid was washed with hexanes, dissolved in dichloromethane, and concentrated three times to yield $2.79 \mathrm{~g}(4.5 \%)$ of $\mathbf{1 3}$ as an orange solid. Formation of $\mathbf{1 3}$ only occurred with new bottles of carbon tetrachloride; distilled carbon tetrachloride did not yield the desired product. ${ }^{1} \mathrm{H}$ NMR $\left(300 \mathrm{MHz}, \mathrm{CDCl}_{3}\right): \delta=1.55-1.69\left(\mathrm{~m}, 2 \mathrm{H}, \mathrm{CH}_{2}\right), 3.32-3.36\left(\mathrm{~m}, 1 \mathrm{H},(\mathrm{CH})_{2}-\right.$ $\left.\mathrm{C} H-\mathrm{CH}_{2}\right), 3.53-3.57\left(\mathrm{~m}, 1 \mathrm{H},(\mathrm{CH})_{2}-\mathrm{CH}-\mathrm{CH}_{2}\right), 3.63-3.68\left(\mathrm{~m}, 2 \mathrm{H},(\mathrm{CH})_{2}-\mathrm{CH}-\mathrm{C}(\mathrm{O})\right), 6.10-6.17(\mathrm{~m}$, $2 \mathrm{H}, H \mathrm{C}=\mathrm{CH}), 14.19(\mathrm{bs}, 1 \mathrm{H}, \mathrm{OH}), 15.12(\mathrm{bs}, 1 \mathrm{H}, \mathrm{C}(\mathrm{O}) \mathrm{OH}) ;{ }^{13} \mathrm{C} \mathrm{NMR}\left(75 \mathrm{MHz}, \mathrm{CDCl}_{3}\right): \delta=$ $47.0\left((\mathrm{CH})_{2}-\mathrm{CH}-\mathrm{CH}_{2}\right), 47.6\left((\mathrm{CH})_{2}-\mathrm{CH}-\mathrm{CH}_{2}\right), 49.5\left(\mathrm{CH}_{2}\right), 49.6\left((\mathrm{CH})_{2}-\mathrm{CH}-\mathrm{C}(\mathrm{O})\right), 50.1\left((\mathrm{CH})_{2}-\right.$ $\mathrm{CH}-\mathrm{C}(\mathrm{O})), 107.3\left((\mathrm{C}(\mathrm{O}))_{2}-\mathrm{C}=\mathrm{C}\right), 135.5(\mathrm{HC}=\mathrm{CH}), 135.9(\mathrm{HC}=\mathrm{CH}), 173.4,175.5,193.2,199.2$; ESI-MS calcd for $\mathrm{C}_{12} \mathrm{H}_{10} \mathrm{O}_{5}[\mathrm{M}-\mathrm{H}]:$ : 233.0450, found 233.0457.

\section{7-Methoxy-5,8-dioxo-1,4,4 $\alpha, 5,8,8 \alpha$-hexahydro-1,4-methanonaphthalene-6- carboxylic acid methyl ester (2):}<smiles>COC(=O)C1=C(OC)C(=O)C2C3C=CC(C2C1=O)C3C1CC1C</smiles>

To a cooled $\left(0{ }^{\circ} \mathrm{C}\right)$, stirred mixture of aqueous $50 \% \mathrm{KOH}(100 \mathrm{~mL})$ and diethyl ether $(200 \mathrm{~mL})$ was added nitrosomethyl urea $(17.6 \mathrm{~g}, 171 \mathrm{mmol})$. When the ether layer turned yellow, it was decanted into an Erlenmeyer flask containing $\mathrm{KOH}$ pellets at $0{ }^{\circ} \mathrm{C}$. The ether layer was then decanted into an ether solution $(200 \mathrm{~mL})$ of $\mathbf{1 3}(2.00 \mathrm{~g}, 8.54 \mathrm{mmol})$ at $0{ }^{\circ} \mathrm{C}$. After $30 \mathrm{~min}$, the reaction mixture was warmed to ambient temperature and stirred for $1 \mathrm{~h}$. Purification was performed using silica gel chromatography (dichloromethane) to yield $1.89 \mathrm{~g}$ (85\%) of 3 as a sticky yellow solid. ${ }^{1} \mathrm{H}$ NMR $\left(300 \mathrm{MHz}, \mathrm{CDCl}_{3}\right): \delta=1.41-1.57\left(\mathrm{~m}, 2 \mathrm{H}, \mathrm{CH}_{2}\right)$, 3.21-3.31 (m, $\left.2 \mathrm{H},(\mathrm{CH})_{2}-\mathrm{CH}-\mathrm{CH}_{2}\right), 3.51-3.54\left(\mathrm{~m}, 2 \mathrm{H},(\mathrm{CH})_{2}-\mathrm{CH}-\mathrm{C}(\mathrm{O})\right), 3.83\left(\mathrm{~s}, 3 \mathrm{H}, \mathrm{CO}-\mathrm{CH}_{3}\right)$, $3.96\left(\mathrm{~s}, 3 \mathrm{H}, \mathrm{C}(\mathrm{O}) \mathrm{O}-\mathrm{CH}_{3}\right), 6.07-6.18(\mathrm{~m}, 2 \mathrm{H}, \mathrm{HC}=\mathrm{CH}) ; ;{ }^{13} \mathrm{C} \mathrm{NMR}\left(75 \mathrm{MHz}, \mathrm{CDCl}_{3}\right): \delta=48.8$ $\left((\mathrm{CH})_{2}-\mathrm{CH}-\mathrm{CH}_{2}\right), 49.0\left((\mathrm{CH})_{2}-\mathrm{CH}-\mathrm{CH}_{2}\right), 49.0\left(\mathrm{CH}_{2}\right), 49.2\left((\mathrm{CH})_{2}-\mathrm{CH}-\mathrm{C}(\mathrm{O})\right), 49.4\left((\mathrm{CH})_{2}-\mathrm{CH}-\right.$ $\mathrm{C}(\mathrm{O})), 52.9\left(\mathrm{CO}-\mathrm{CH}_{3}\right), 59.8\left(\mathrm{C}(\mathrm{O}) \mathrm{O}-\mathrm{CH}_{3}\right), 135.0(\mathrm{HC}=\mathrm{CH}), 136.1(\mathrm{HC}=\mathrm{CH}), 158.9,164.7$, 194.8, 195.4; ESI-MS calcd for $\mathrm{C}_{14} \mathrm{H}_{14} \mathrm{O}_{5}[\mathrm{M}+\mathrm{H}]^{+}:$: 263.0919, found 263.0929. 
5,7,8-Trimethoxy-1,4-dihydro-1,4-methanonaphthalene-6-carboxylic acid methyl ester (14):<smiles>COC(=O)c1c(O)c(OC)c2c(c1OC)C1C=CC2C1</smiles>

To a refluxing solution of $2(2.50 \mathrm{~g}, 9.53 \mathrm{mmol})$ in acetone $(60 \mathrm{~mL})$ was added dimethyl sulfate $(9.0 \mathrm{~mL}, 95 \mathrm{mmol})$. A $10 \%$ methanolic $\mathrm{KOH}$ solution was added dropwise until the color of the solution stopped changing from purple to tan. The reaction mixture was heated at reflux for $1 \mathrm{~h}$, at which point a $2 \mathrm{~N} \mathrm{HCl}$ solution was added until the mixture turned clear. The reaction mixture was extracted four times with dichloromethane, dried over sodium sulfate, and concentrated. Purification was performed using silica gel chromatography (1:4 ethyl acetate/hexanes) to yield $1.86 \mathrm{~g}(67 \%)$ of 14 as a yellow oil. ${ }^{1} \mathrm{H} \mathrm{NMR}\left(300 \mathrm{MHz}, \mathrm{CDCl}_{3}\right): \delta=$ 2.15-2.23 (m, 2H, CH $), 3.83\left(\mathrm{~s}, 3 \mathrm{H}, \mathrm{O}-\mathrm{CH}_{3}\right), 3.83\left(\mathrm{~s}, 3 \mathrm{H}, \mathrm{O}-\mathrm{CH}_{3}\right), 3.85\left(\mathrm{~s}, 3 \mathrm{H}, \mathrm{O}-\mathrm{CH}_{3}\right), 3.90(\mathrm{~s}$, $\left.3 \mathrm{H}, \mathrm{CO}_{2}-\mathrm{CH}_{3}\right), 4.16-4.18\left(\mathrm{~m}, 2 \mathrm{H}, \mathrm{CH}-\mathrm{CH}(\mathrm{C})-\mathrm{CH}_{2}\right), 6.76-6.82(\mathrm{~m}, 2 \mathrm{H}, \mathrm{HC}=\mathrm{CH}) ;{ }^{13} \mathrm{C}$ NMR $(75$ $\left.\mathrm{MHz}, \mathrm{CDCl}_{3}\right): \delta=47.6\left(\mathrm{CH}-\mathrm{CH}(\mathrm{C})-\mathrm{CH}_{2}\right), 48.0\left(\mathrm{CH}-\mathrm{CH}(\mathrm{C})-\mathrm{CH}_{2}\right), 52.5\left(\mathrm{CO}_{2}-\mathrm{CH}_{3}\right), 61.6(\mathrm{O}-$ $\left.\mathrm{CH}_{3}\right), 61.9\left(\mathrm{O}-\mathrm{CH}_{3}\right), 62.2\left(\mathrm{O}-\mathrm{CH}_{3}\right), 68.5\left(\mathrm{CH}_{2}\right), 119.2,137.2,142.5(\mathrm{HC}=\mathrm{CH}), 142.8(\mathrm{HC}=\mathrm{CH})$, 144.0, 146.6, 146.8, 147.9, 167.0; TLC: $\mathrm{R}_{\mathrm{f}}=0.67$ (2:1 ethyl acetate/hexanes); ESI-MS calcd for $\mathrm{C}_{16} \mathrm{H}_{18} \mathrm{O}_{5}[\mathrm{M}+\mathrm{Na}]^{+}:$313.1052, found 313.1037.

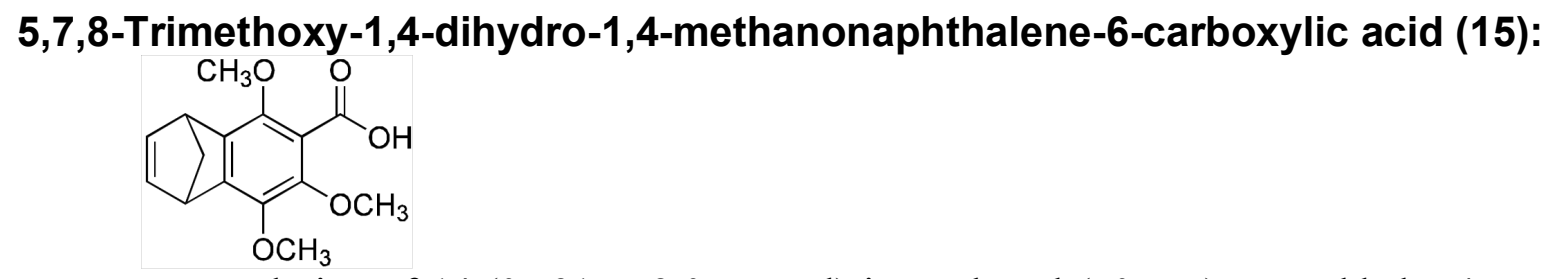

To a solution of $14(0.591 \mathrm{~g}, 2.05 \mathrm{mmol})$ in methanol $(50 \mathrm{~mL})$ was added a $4 \mathrm{~N} \mathrm{NaOH}$ solution $(50 \mathrm{~mL})$, and the resulting reaction mixture was heated at reflux for $5 \mathrm{~h}$. Methanol was removed under reduced pressure and a $3 \mathrm{~N} \mathrm{HCl}$ solution was added to achieve $\mathrm{pH} 1$. The reaction mixture was extracted three times with ethyl acetate, dried over sodium sulfate, and concentrated under reduced pressure to yield $0.567 \mathrm{~g}$ of $\mathbf{1 5}$ as a brown oil in nearly quantitative yield. ${ }^{1} \mathrm{H}$ NMR $\left(300 \mathrm{MHz}, \mathrm{CDCl}_{3}\right): \delta=2.18-2.27\left(\mathrm{~m}, 2 \mathrm{H}, \mathrm{CH}_{2}\right), 3.86\left(\mathrm{~s}, 3 \mathrm{H}, \mathrm{O}-\mathrm{CH}_{3}\right), 3.89(\mathrm{~s}$, $\left.3 \mathrm{H}, \mathrm{O}-\mathrm{CH}_{3}\right), 3.92\left(\mathrm{~s}, 3 \mathrm{H}, \mathrm{O}-\mathrm{CH}_{3}\right), 4.19-4.22\left(\mathrm{~m}, 2 \mathrm{H}, \mathrm{CH}-\mathrm{CH}(\mathrm{C})-\mathrm{CH}_{2}\right), 6.78-6.86(\mathrm{~m}, 2 \mathrm{H}$, $H \mathrm{C}=\mathrm{CH}) ;{ }^{13} \mathrm{C} \mathrm{NMR}\left(75 \mathrm{MHz}, \mathrm{CDCl}_{3}\right): \delta=47.6\left(\mathrm{CH}-\mathrm{CH}(\mathrm{C})-\mathrm{CH}_{2}\right), 47.9\left(\mathrm{CH}-\mathrm{CH}(\mathrm{C})-\mathrm{CH}_{2}\right), 61.6$ $\left(\mathrm{CH}_{3}\right), 62.1\left(\mathrm{CH}_{3}\right), 62.5\left(\mathrm{CH}_{3}\right), 68.5\left(\mathrm{CH}_{2}\right), 117.9,137.8,142.4(\mathrm{HC}=\mathrm{CH}), 142.9(\mathrm{HC}=\mathrm{CH})$, 144.2, 147.2, 147.6, 148.6, 170.6; ESI-MS calcd for $\mathrm{C}_{15} \mathrm{H}_{16} \mathrm{O}_{5}[\mathrm{M}-\mathrm{H}]$ : 275.0920 , found 275.0910 .

\section{5,7,8-Trimethoxy-1,4-dihydro-1,4-methanonaphthalene-6-carboxylic acid 2,5- dioxopyrrolidin-1-yl ester (3):}<smiles>COc1c(OC)c2c(c(O)c1C(=O)ON1C(=O)CCC1=O)C1C=CC2C1</smiles>

To a solution of $15(0.972 \mathrm{~g}, 3.52 \mathrm{mmol})$ in dichloromethane $(80 \mathrm{~mL})$ was added $\mathrm{N}$ hydroxysuccinimide $(0.547 \mathrm{~g}, 4.75 \mathrm{mmol})$ and 1-[3-(dimethylamino)propyl]-3-ethylcarbodiimide 
hydrochloride $(0.890 \mathrm{~g}, 4.64 \mathrm{mmol})$, and the resulting reaction mixture was stirred under dinitrogen for $15 \mathrm{~h}$. The reaction mixture was diluted with dichloromethane $(100 \mathrm{~mL})$, washed twice with a $0.01 \mathrm{~N} \mathrm{HCl}$ solution $(50 \mathrm{~mL})$, dried over sodium sulfate, and concentrated under reduced pressure. Purification was performed using silica gel chromatography (dichloromethane $\rightarrow$ 2:1 ethyl acetate/hexanes) to yield $0.898 \mathrm{~g}(68 \%)$ of 3 as a colorless sticky solid. ${ }^{1} \mathrm{H}$ NMR $\left(300 \mathrm{MHz}, \mathrm{CDCl}_{3}\right): \delta=2.19-2.27\left(\mathrm{~m}, 2 \mathrm{H}, \mathrm{CH}-\mathrm{CH}_{2}-\mathrm{CH}\right), 2.89\left(\mathrm{bs}, 4 \mathrm{H}, \mathrm{CH}_{2}-\mathrm{CH}_{2}\right), 3.86(\mathrm{~s}, 3 \mathrm{H}$, $\left.\mathrm{O}-\mathrm{CH}_{3}\right), 3.92$ (s, 3H, O-CH $), 3.95\left(\mathrm{~s}, 3 \mathrm{H}, \mathrm{O}-\mathrm{CH}_{3}\right), 4.19-4.23$ (m, 2H, CH-CH(C)-CH $), 6.78-$ $6.85(\mathrm{~m}, 2 \mathrm{H}, \mathrm{HC}=\mathrm{CH}) ;{ }^{13} \mathrm{C}$ NMR $\left(75 \mathrm{MHz}, \mathrm{CDCl}_{3}\right): \delta=25.9\left(\mathrm{CH}_{2}-\mathrm{CH}_{2}\right), 47.7(\mathrm{CH}-\mathrm{CH}(\mathrm{C})-$ $\left.\mathrm{CH}_{2}\right), 47.9\left(\mathrm{CH}-\mathrm{CH}(\mathrm{C})-\mathrm{CH}_{2}\right), 61.7\left(\mathrm{O}-\mathrm{CH}_{3}\right), 62.3\left(\mathrm{O}-\mathrm{CH}_{3}\right), 62.6\left(\mathrm{O}-\mathrm{CH}_{3}\right), 68.6\left(\mathrm{CH}-\mathrm{CH}_{2}-\mathrm{CH}\right)$, 137.7, $142.4(\mathrm{CH}=\mathrm{CH}), 143.0(\mathrm{CH}=\mathrm{CH}), 144.2,147.9,149.6,149.9,161.4,169.1 ;$ TLC: $\mathrm{R}_{\mathrm{f}}=$ 0.42 (2:1 ethyl acetate/hexanes); ESI-MS calcd for $\mathrm{C}_{19} \mathrm{H}_{19} \mathrm{NO}_{7}[\mathrm{M}+\mathrm{Na}]^{+}: 396.1059$, found 396.1069 .

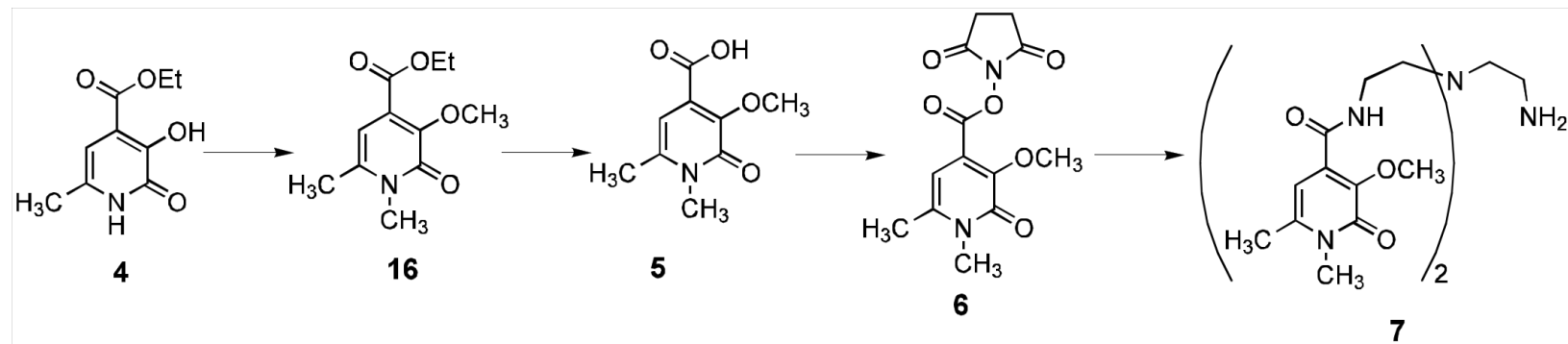

3-Methoxy-1,6-dimethyl-2-oxo-1,2-dihydropyridine-4-carboxylic acid ethyl ester (16):<smiles>CCOC(=O)c1cc(C)n(C)c(=O)c1OC</smiles>

To a solution of 4 (8.69 $\mathrm{g}, 44.1 \mathrm{mmol})$ in dimethylformamide (440 $\mathrm{mL})$ was added potassium carbonate $(12.7 \mathrm{~g}, 91.6 \mathrm{mmol})$ and methyl iodide $(27.5 \mathrm{~mL}, 441 \mathrm{mmol})$, and the resulting reaction mixture was stirred for $15 \mathrm{~h}$. The reaction was concentrated under reduced pressure and diluted with dichloromethane $(200 \mathrm{~mL})$ and water $(200 \mathrm{~mL})$. The layers were separated, and the aqueous layer was extracted with dichloromethane $(50 \mathrm{~mL})$. The combined organic layers were dried over sodium sulfate and concentrated under reduced pressure. Purification was performed using silica gel chromatography (2:1 ethyl acetate/hexanes) to yield $7.89 \mathrm{~g}(80 \%)$ of 16 as a tan solid. ${ }^{1} \mathrm{H} \mathrm{NMR}\left(300 \mathrm{MHz}, \mathrm{CDCl}_{3}\right): \delta=1.36\left(\mathrm{t}, 3 \mathrm{H}, J=7.1 \mathrm{~Hz}, \mathrm{CH}_{2^{-}}\right.$ $\left.\mathrm{CH}_{3}\right), 2.32$ (s, 3H, C-CH $), 3.52\left(\mathrm{~s}, 3 \mathrm{H}, \mathrm{N}-\mathrm{CH} H_{3}\right), 3.94$ (s, 3H, O-CH $), 4.33$ (q, 2H, J=7.1 Hz, $\left.\mathrm{CH}_{2}\right), 6.16(\mathrm{~s}, 1 \mathrm{H}, \mathrm{CH}) ;{ }^{13} \mathrm{C}$ NMR $\left(75 \mathrm{MHz}, \mathrm{CDCl}_{3}\right): \delta=14.3\left(\mathrm{CH}_{2}-\mathrm{CH}_{3}\right), 20.7\left(\mathrm{C}-\mathrm{CH}_{3}\right), 31.9$ $\left(\mathrm{N}-\mathrm{CH}_{3}\right), 60.4\left(\mathrm{O}-\mathrm{CH}_{3}\right), 61.7\left(\mathrm{CH}_{2}\right), 103.8(\mathrm{CH}), 129.5,140.3,146.4,160.7,165.4$; TLC: $\mathrm{R}_{\mathrm{f}}=$ 0.20 (2:1 ethyl acetate/hexanes); ESI-MS calcd for $\mathrm{C}_{11} \mathrm{H}_{15} \mathrm{NO}_{4}[\mathrm{M}+\mathrm{H}]^{+}:$226.1079, found 226.1072 . 
3-Methoxy-1,6-dimethyl-2-oxo-1,2-dihydropyridine-4-carboxylic acid (5):<smiles>COc1c(C(=O)O)cc(C)n(C)c1=O</smiles>

To a solution of $\mathbf{1 6}(7.51 \mathrm{~g}, 33.3 \mathrm{mmol})$ in methanol $(350 \mathrm{~mL})$ was added a $4 \mathrm{M}$ sodium hydroxide solution $(350 \mathrm{~mL})$. The resulting solution was heated at reflux, and after $2 \mathrm{~h}$, methanol was removed under reduced pressure. An aqueous $3 \mathrm{~N} \mathrm{HCl}$ solution was used to adjust the resulting solution to $\mathrm{pH} 1$. The solution was extracted with ethyl acetate; the organic extracts were dried over sodium sulfate, and solvent was removed under reduced pressure to yield $6.44 \mathrm{~g}$ $(98 \%)$ of 5 as a tan solid. ${ }^{1} \mathrm{H}$ NMR $\left(300 \mathrm{MHz}, \mathrm{CD}_{3} \mathrm{OD}\right): \delta=2.40\left(\mathrm{~s}, 3 \mathrm{H}, \mathrm{C}-\mathrm{CH}_{3}\right), 3.58(\mathrm{~s}, 3 \mathrm{H}$, $\left.\mathrm{N}-\mathrm{CH}_{3}\right), 3.86\left(\mathrm{~s}, 3 \mathrm{H}, \mathrm{O}-\mathrm{CH}_{3}\right), 6.36(\mathrm{~s}, 1 \mathrm{H}, \mathrm{CH}) ;{ }^{13} \mathrm{C} \mathrm{NMR}\left(75 \mathrm{MHz}, \mathrm{CD}_{3} \mathrm{OD}\right): \delta=20.6(\mathrm{C}-$ $\left.\mathrm{CH}_{3}\right), 32.6\left(\mathrm{~N}-\mathrm{CH}_{3}\right), 61.0\left(\mathrm{O}-\mathrm{CH}_{3}\right), 105.9(\mathrm{CH}), 132.8,143.4,146.6,162.6$, 168.2; ESI-MS calcd for $\mathrm{C}_{9} \mathrm{H}_{11} \mathrm{NO}_{4}[\mathrm{M}-\mathrm{H}]^{-}:$196.0610, found 196.0615 .

\section{3-Methoxy-1,6-dimethyl-2-oxo-1,2-dihydropyridine-4-carboxylic acid 2,5-} dioxopyrrolidin-1-yl ester (6):<smiles>COc1c(C(=O)ON2C(=O)CCC2=O)cc(C)n(C)c1=O</smiles>

To a solution of $5(5.36 \mathrm{~g}, 27.2 \mathrm{mmol})$ in dichloromethane $(500 \mathrm{~mL})$ was added $\mathrm{N}$ hydroxysuccinimide (4.23 g, $36.7 \mathrm{mmol})$ and 1-[3-(dimethylamino)propyl]-3-ethylcarbodiimide hydrochloride $(6.89 \mathrm{~g}, 35.9 \mathrm{mmol})$, and the resulting reaction mixture was stirred under dinitrogen for $15 \mathrm{~h}$. The reaction mixture was washed twice with a $0.01 \mathrm{~N} \mathrm{HCl}$ solution (150 $\mathrm{mL}$ ), dried over sodium sulfate, and concentrated under reduced pressure. Purification was performed using silica gel chromatography (1:49 methanol/dichloromethane) to yield $7.83 \mathrm{~g}$ (98\%) of 6 as a tan solid. ${ }^{1} \mathrm{H}$ NMR $\left(300 \mathrm{MHz}, \mathrm{CDCl}_{3}\right): \delta=2.31\left(\mathrm{~s}, 3 \mathrm{H}, \mathrm{C}-\mathrm{CH}_{3}\right), 4.08(\mathrm{~s}, 4 \mathrm{H}$, $\left.\mathrm{CH}_{2}\right), 3.50$ (s, 3H, N-CH $), 3.98$ (s, 3H, O-CH $), 6.29$ (s, $\left.1 \mathrm{H}, \mathrm{CH}\right) ;{ }^{13} \mathrm{C} \mathrm{NMR}\left(75 \mathrm{MHz}, \mathrm{CDCl}_{3}\right)$ : $\delta=20.6\left(\mathrm{C}-\mathrm{CH}_{3}\right), 25.8\left(\mathrm{CH}_{2}\right), 32.0\left(\mathrm{~N}-\mathrm{CH}_{3}\right), 60.8\left(\mathrm{O}-\mathrm{CH}_{3}\right), 103.0(\mathrm{CH}), 122.8,140.7,148.8$, 159.9, 160.3, 169.0; TLC: $\mathrm{R}_{\mathrm{f}}=0.33$ (1:19 methanol/dichloromethane); ESI-MS calcd for $\mathrm{C}_{13} \mathrm{H}_{14} \mathrm{~N}_{2} \mathrm{O}_{6}[\mathrm{M}+\mathrm{H}]^{+}:$295.0930, found 295.0931.

\section{Compound 7:}

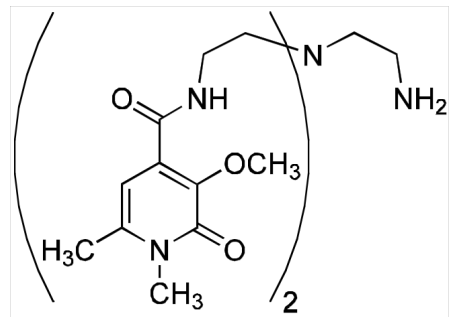

Tritylchloride resin $(2.0 \mathrm{~g}, 3.2 \mathrm{mmol})$ was swelled in tetrahydrofuran $(20 \mathrm{~mL})$ for $15 \mathrm{~min}$ at which point tris(2-aminoethyl)amine $(4.98 \mathrm{~mL}, 32.0 \mathrm{mmol})$ was added, and the resulting 
solution was mixed for $20 \mathrm{~h}$. The resin was drained and washed four times with $20 \mathrm{~mL}$ of 17:2:1 dichloromethane/methanol/diisopropylethylamine, and three times with $20 \mathrm{~mL}$ of dichloromethane. To the washed resin was added dichloromethane $(20 \mathrm{~mL})$, diisopropylethylamine $(5.57 \mathrm{~mL}, 32.0 \mathrm{mmol})$, and $6(3.77 \mathrm{~g}, 12.8 \mathrm{mmol})$, and the resulting solution was stirred for $20 \mathrm{~h}$. The resin was drained and washed three times with $20 \mathrm{~mL}$ of dichloromethane and dried under reduced pressure. A solution of 38:1:1 trifluoroacetic acid/triisopropylsilane/water $(24 \mathrm{~mL})$ was added to the resin; after $2 \mathrm{~h}$, the resin was filtered and rinsed with trifluoroacetic acid $(5 \mathrm{~mL})$. The combined trifluoroacetic acid-containing filtrate was reduced in volume to $2 \mathrm{~mL}$. This solution was added dropwise to diethyl ether $(400 \mathrm{~mL})$ at $0{ }^{\circ} \mathrm{C}$, and the resulting precipitate was collected. Purification was performed using basic alumina chromatography $(1: 9 \rightarrow 3: 7$ methanol/dichloromethane) to yield $1.0 \mathrm{~g}(63 \%)$ of 7 as a white solid. ${ }^{1} \mathrm{H}$ NMR (300 MHz, $\left.\mathrm{CD}_{3} \mathrm{OD}\right): \delta=2.39\left(\mathrm{~s}, 6 \mathrm{H}, \mathrm{C}-\mathrm{CH}_{3}\right), 2.62-2.78\left(\mathrm{~m}, 8 \mathrm{H}, \mathrm{H}_{2} \mathrm{~N}^{-\mathrm{CH}_{2}}\right.$ and $\left.\mathrm{N}-\left(\mathrm{CH}_{2}\right)_{3}\right), 3.48\left(\mathrm{t}, 4 \mathrm{H}, \mathrm{J}=6.2 \mathrm{~Hz}, \mathrm{C}(\mathrm{O}) \mathrm{NH}-\mathrm{CH}_{2}\right), 3.56\left(\mathrm{~s}, 6 \mathrm{H}, \mathrm{N}-\mathrm{CH}_{3}\right), 3.88\left(\mathrm{~s}, 6 \mathrm{H}, \mathrm{O}-\mathrm{CH}_{3}\right)$, $6.40(\mathrm{~s}, 2 \mathrm{H}, \mathrm{CH}) ;{ }^{13} \mathrm{C}$ NMR $\left(75 \mathrm{MHz}, \mathrm{CD}_{3} \mathrm{OD}\right): \delta=20.7\left(\mathrm{C}-\mathrm{CH}_{3}\right), 32.6\left(\mathrm{~N}-\mathrm{CH}_{3}\right), 39.1$ $\left(\mathrm{C}(\mathrm{O}) \mathrm{NH}-\mathrm{CH}_{2}\right), 40.3\left(\mathrm{H}_{2} \mathrm{~N}-\mathrm{CH}_{2}\right), 54.5\left(\mathrm{~N}-\left(\mathrm{CH}_{2}\right)_{3}\right), 57.7\left(\mathrm{~N}-\left(\mathrm{CH}_{2}\right)_{3}\right), 60.9\left(\mathrm{O}-\mathrm{CH}_{3}\right), 105.7(\mathrm{CH})$, 133.3, 143.3, 145.5, 161.9, 166.4; TLC: $\mathrm{R}_{\mathrm{f}}=0.21$ (1:19 methanol/dichloromethane on basic alumina plates); ESI-MS calcd for $\mathrm{C}_{24} \mathrm{H}_{36} \mathrm{~N}_{6} \mathrm{O}_{6}[\mathrm{M}+\mathrm{H}]^{+}$: 505.2775, found 505.2794.

\section{General Procedure for Polymerization Reactions:}
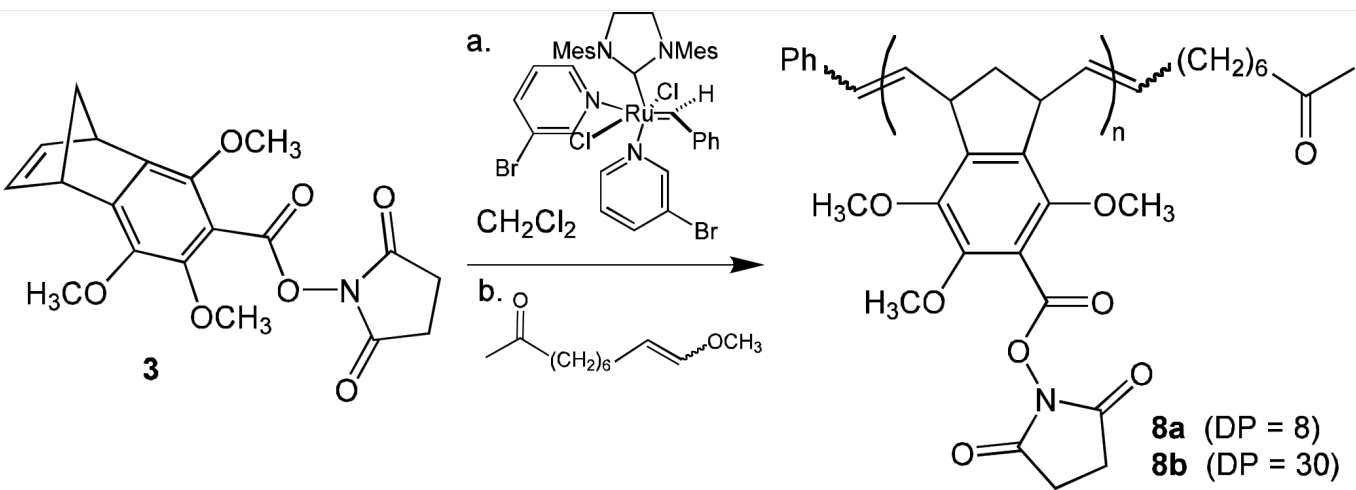

Polymerizations were carried out under an argon atmosphere in scintillation vials. Solutions of $\left(\mathrm{H}_{2} \mathrm{IMes}\right)(3-\mathrm{Br}-\mathrm{py})_{2}(\mathrm{Cl})_{2} \mathrm{Ru}=\mathrm{CHPh}(5.35 \mathrm{mM})$ and $3(0.134 \mathrm{M})$ in degassed dichloromethane were cooled to $-20{ }^{\circ} \mathrm{C}$. The solutions were combined in the desired monomer to initiator ratio, and degassed dichloromethane was added to bring the final concentration of $\mathbf{3}$ to $38.3 \mathrm{mM}$. The reactions were allowed to warm slowly to ambient temperature. After consumption of $\mathbf{3}$, as determined by TLC, 10-methoxydec-9-en-2-one ( $20 \mu \mathrm{L}, 0.10 \mathrm{mmol})$ was added, and the reaction mixture was allowed to stir for $15 \mathrm{~h}$. The reaction mixtures were added dropwise to a 30 -fold volume excess of diethyl ether. The resulting white solid was collected, and residual solvent was removed under reduced pressure. 


\section{Polymer 8a:}<smiles>COc1c(OC)c(C(=O)ON2C(=O)CCC2=O)c2c(c1OC)C(CC(C)(C)CCCC(C)=O)CC2CC(C)(C)C(C)=O</smiles>

Yield $=76.3 \mathrm{mg}(71 \%)$ black solid. ${ }^{1} \mathrm{H}$ NMR $\left(500 \mathrm{MHz}\right.$, DMSO- $\left.d_{6}\right): \delta=1.53(\mathrm{bs}), 2.50$ (bs), 2.86 (bs, $\mathrm{C}(=\mathrm{O})-\mathrm{CH}_{2}-\mathrm{CH}_{2}-\mathrm{C}(=\mathrm{O})$ ), 3.73 (bs, $\left.\mathrm{O}-\mathrm{CH}_{3}\right), 4.24$ (bs), 5.52 (bs, $18 \mathrm{H}, H \mathrm{C}=\mathrm{CH}$ ), 7.14 (bs, 5H, $\mathrm{C}_{6} H_{5}$ ); PDI 1.58; Calculated MW 3217; $\mathrm{M}_{\mathrm{w}} 2788 ; \mathrm{M}_{\mathrm{n}} 1760$.

\section{Polymer 8b:}

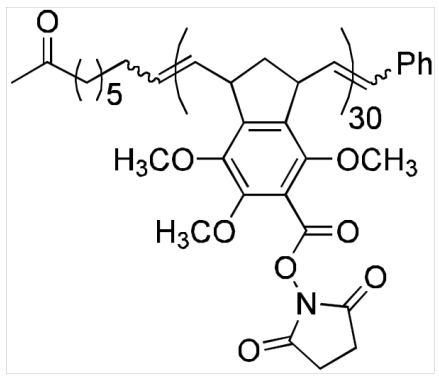

Yield $=165 \mathrm{mg}(94 \%)$ off-white solid. ${ }^{1} \mathrm{H}$ NMR $\left(500 \mathrm{MHz}, \mathrm{DMSO}-d_{6}\right): \delta=1.07$ (bs), 2.84 (bs, $\mathrm{C}(=\mathrm{O})-\mathrm{CH}_{2}-\mathrm{CH}_{2}-\mathrm{C}(=\mathrm{O})$ ), 3.64 (bs, $\left.\mathrm{O}-\mathrm{CH}_{3}\right), 5.31$ (bs, 63H, $\left.H \mathrm{C}=\mathrm{CH}\right), 7.00$ (bs, 5H, $\left.\mathrm{C}_{6} H_{5}\right)$; PDI 1.21; Calculated MW 11431; $\mathrm{M}_{\mathrm{w}} 5004 ; \mathrm{M}_{\mathrm{n}} 4122$.

\section{General Procedure for Conjugation of 8 with 7 and 14:}
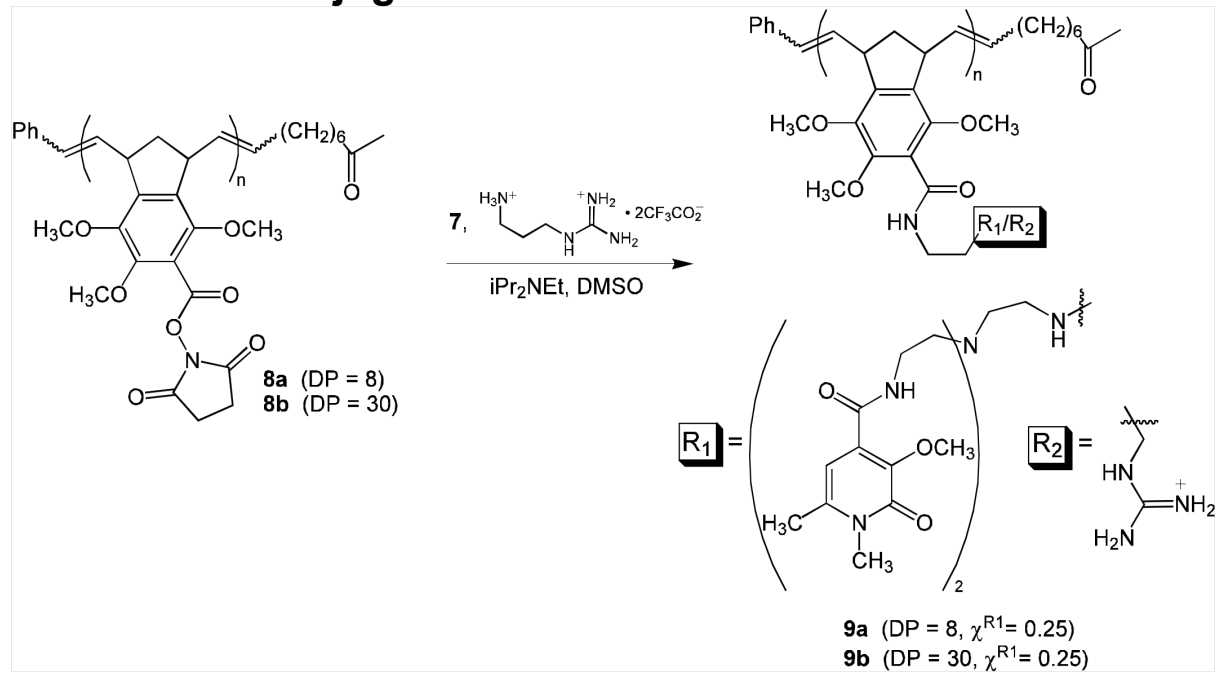

To a solution of $8(5 \mathrm{mg})$ in dimethylsulfoxide $(200 \mu \mathrm{L})$ and diisopropylethylamine (10 equivalents per monomer unit) was added 7 (0.25 equivalents per monomer unit) and $\mathrm{N}$-(3aminopropyl)guanidine bis-trifluoroacetic acid salt ( 0.75 equivalents per monomer unit). After $15 \mathrm{~h}$, the entire reaction mixture was passed through a PD-10 column. Fractions containing polymer were collected, and solvent was removed under reduced pressure. 


\section{Polymer 9a:}

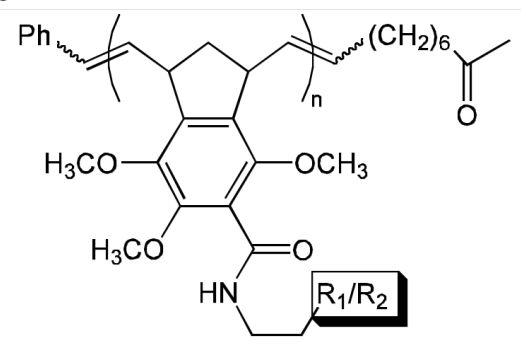<smiles>CCN(CCNC(=O)c1cc(C)n(C)c(=O)c1OC)C(C)C</smiles>

$$
\left(\mathrm{DP}=8, \chi^{\mathrm{R} 1}=0.25\right)
$$

Yield $=12.4 \mathrm{mg}(70 \%)$ brown solid. ${ }^{1} \mathrm{H}$ NMR (500 MHz, DMSO- $\left.d_{6}\right): \delta=1.18(\mathrm{bs}), 1.68$ (bs), 2.31 (bs), 2.65 (bs), 3.20 (bs), 3.43 (bs), 3.68 (bs), 4.12 (bs), 5.42 (bs, $17 \mathrm{H}, H \mathrm{C}=\mathrm{CH}$ ), 6.22 (bs, 2.9H, C-CH=C-CH ), 7.20 (bs), 7.69 (bs), 8.31 (bs).

\section{Polymer 9b:}

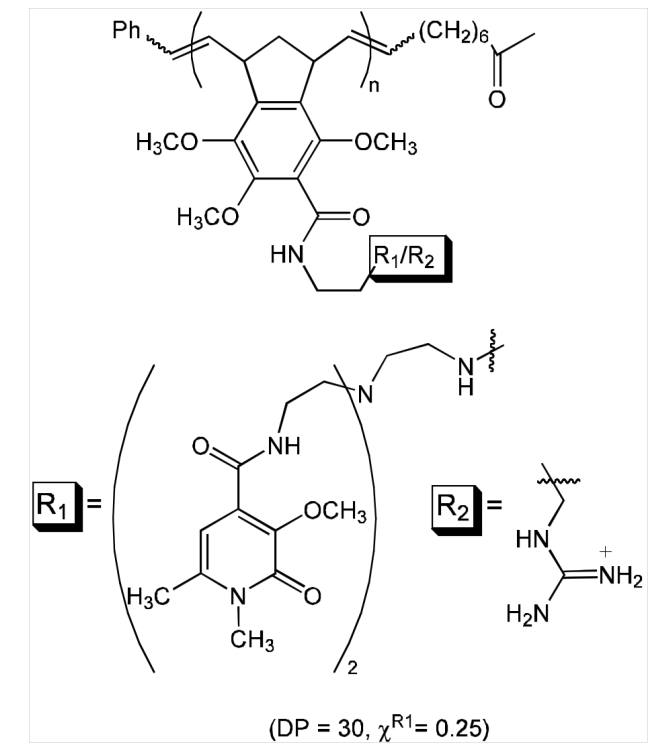

Yield $=8.37 \mathrm{mg}(38 \%)$ brown glass. ${ }^{1} \mathrm{H}$ NMR $\left(500 \mathrm{MHz}\right.$, DMSO- $\left.d_{6}\right): \delta=1.18(\mathrm{bs}), 1.68$ (bs), 2.31 (bs), 2.65 (bs), 3.20 (bs), 3.43 (bs), 3.68 (bs), 4.12 (bs), 5.42 (bs, 62H, $H \mathrm{C}=\mathrm{CH}), 6.22$ (bs, 7.6H, C-CH=C-CH ), 7.20 (bs), 7.69 (bs), 8.31 (bs). 


\section{Compound 12:}

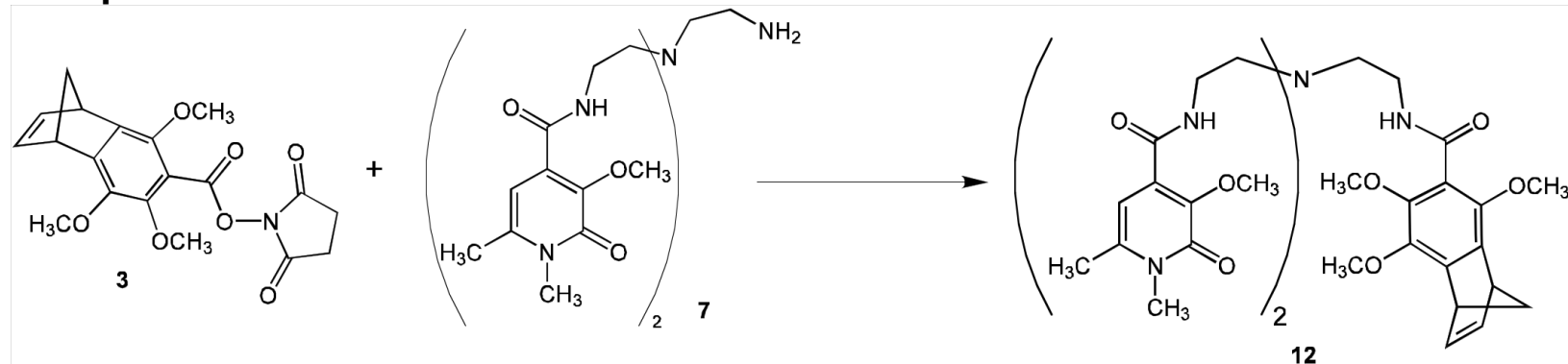

A solution of $7(0.304 \mathrm{~g}, 0.603 \mathrm{mmol}), 3(0.150 \mathrm{~g}, 0.402 \mathrm{mmol})$, and diisopropylethylamine $(0.350 \mathrm{~mL}, 2.01 \mathrm{mmol})$ in dichloromethane $(10 \mathrm{~mL})$ was stirred under dinitrogen for $4 \mathrm{~h}$, at which point solvent was removed under reduced pressure. Purification was performed using silica gel chromatography (1:19 methanol/dichloromethane) to yield $0.202 \mathrm{~g}$ $(66 \%)$ of 12 as a white solid. ${ }^{1} \mathrm{H}$ NMR $\left(300 \mathrm{MHz}_{\mathrm{CDCl}}\right)$ : $\delta=2.10-2.20\left(\mathrm{~m}, 2 \mathrm{H}, \mathrm{CH}-\mathrm{CH} \mathrm{C}_{2}-\mathrm{CH}\right)$, $2.33\left(\mathrm{~s}, 6 \mathrm{H}, \mathrm{C}-\mathrm{CH}_{3}\right), 2.79-2.86\left(\mathrm{~m}, 6 \mathrm{H}, \mathrm{N}-\left(\mathrm{CH}_{2}\right)_{3}\right), 3.49-3.55\left(\mathrm{~m}, 12 \mathrm{H}, \mathrm{C}(\mathrm{O}) \mathrm{NH}-\mathrm{CH}_{2}\right.$ and N$\left.\mathrm{CH}_{3}\right), 3.80\left(\mathrm{~s}, 3 \mathrm{H}, \mathrm{O}-\mathrm{CH}_{3}\right), 3.81\left(\mathrm{~s}, 3 \mathrm{H}, \mathrm{O}-\mathrm{CH}_{3}\right), 3.82\left(\mathrm{~s}, 3 \mathrm{H}, \mathrm{O}-\mathrm{CH}_{3}\right), 3.94(\mathrm{~s}, 6 \mathrm{H}, \mathrm{O}=\mathrm{C}-\mathrm{C}(=\mathrm{C})-$ $\left.\mathrm{O}-\mathrm{CH}_{3}\right), 4.11-4.14\left(\mathrm{~m}, 2 \mathrm{H}, \mathrm{CH}-\mathrm{CH}(\mathrm{C})-\mathrm{CH}_{2}\right), 6.27-6.31\left(\mathrm{t}, 1 \mathrm{H}, J=5.6 \mathrm{~Hz},\left(\mathrm{CH}_{3}-\mathrm{O}-\mathrm{C}\right)_{2}-\mathrm{C}-\right.$ $\mathrm{C}(=\mathrm{O})-\mathrm{NH}), 6.55\left(\mathrm{~s}, 2 \mathrm{H}, \mathrm{CH}_{3}-\mathrm{C}=\mathrm{CH}-\mathrm{C}\right), 6.73-6.78(\mathrm{~m}, 2 \mathrm{H}, \mathrm{CH}=\mathrm{CH}), 8.16-8.20(\mathrm{t}, 2 \mathrm{H}, J=5.2$ $\mathrm{Hz}, \mathrm{CH}-\mathrm{C}-\mathrm{C}(=\mathrm{O})-\mathrm{NH}) ;{ }^{13} \mathrm{C} \mathrm{NMR}\left(75 \mathrm{MHz}, \mathrm{CDCl}_{3}\right): \delta=20.8\left(\mathrm{C}-\mathrm{CH}_{3}\right), 31.9\left(\mathrm{~N}-\mathrm{CH}_{3}\right), 38.2$ $\left(\mathrm{C}(\mathrm{O}) \mathrm{NH}-\mathrm{CH}_{2}\right), 47.6\left(\mathrm{CH}-\mathrm{CH}(\mathrm{C})-\mathrm{CH}_{2}\right), 47.9\left(\mathrm{CH}-\mathrm{CH}(\mathrm{C})-\mathrm{CH}_{2}\right), 53.7\left(\mathrm{~N}-\left(\mathrm{CH}_{2}\right)_{3}\right), 53.8(\mathrm{~N}-$ $\left.\left(\mathrm{CH}_{2}\right)_{3}\right), 60.0\left(\mathrm{O}=\mathrm{C}-\mathrm{C}(=\mathrm{C})-\mathrm{O}-\mathrm{CH}_{3}\right), 61.6\left(\mathrm{O}-\mathrm{CH}_{3}\right), 62.1\left(\mathrm{O}-\mathrm{CH}_{3}\right), 62.4\left(\mathrm{O}-\mathrm{CH}_{3}\right), 68.4\left(\mathrm{CH}-\mathrm{CH}_{2}-\right.$ $\mathrm{CH}), 104.5\left(\mathrm{CH}_{3}-\mathrm{C}=\mathrm{CH}-\mathrm{C}\right), 122.3,129.3,137.4,140.2,142.4(\mathrm{CH}=\mathrm{CH}), 142.9(\mathrm{CH}=\mathrm{CH})$, $144.2, \quad 145.1, \quad 145.7, \quad 146.9, \quad 147.7, \quad 160.2, \quad 163.9, \quad 166.2 ; \quad T L C: \quad R_{f}=0.26 \quad(2: 23$ methanol/dichloromethane); ESI-MS calcd for $\mathrm{C}_{39} \mathrm{H}_{50} \mathrm{~N}_{6} \mathrm{O}_{10}[\mathrm{M}+\mathrm{H}]^{+}$: 763.3667, found 763.3648 .

\section{General Procedure for Deprotection of Methyl Ethers and Metallation:}

To a solution of methyl ether-protected chelate (1 eq. of $9 \mathbf{a}, 9 \mathbf{b}$, or 12 ) in anhydrous dimethylsulfoxide (DMSO) under argon was added a solution of sodium ethanethiolate (2.5 eq. per $\mathrm{OMe}$ ) in anhydrous DMSO to make a final solution of between 0.4 and $26 \mathrm{mM}$ chelate and between 44 and $328 \mathrm{mM}$ sodium ethanethiolate. The resulting solution was heated to $142{ }^{\circ} \mathrm{C}$ for $1.5 \mathrm{~h}$ at which point a four-fold volume excess of water was added to quench excess sodium ethanethiolate. The reaction mixture was concentrated to dryness under reduced pressure, and LC-MS of the product resulting from 12 showed one major product with a mass corresponding to four of five methyl ethers removed.

To ensure that the remaining methoxy group was not in a position to interfere with metal chelation, a portion of the intermediate $(0.80 \mathrm{mg}, 1.2 \mu \mathrm{mol})$ was dissolved in anhydrous DMSO $(11.4 \mu \mathrm{L})$ under argon. To the resulting solution was added $\mathrm{CH}_{2} \mathrm{I}_{2}(1.4 \mu \mathrm{mol}, 0.11 \mu \mathrm{L})$ and sodium carbonate $(2.5 \mu \mathrm{mol}, 0.27 \mathrm{mg})$, and the reaction was heated to $55{ }^{\circ} \mathrm{C}$ for $8 \mathrm{~h}$. The mixture was cooled to ambient temperature, and $2 \mathrm{~mL}$ of water was added. LC/MS indicated that there was one major product with mass $\left([\mathrm{M}+\mathrm{H}]^{+}=721.4\right)$ corresponding to the formation of a methylene bridge between the two ortho hydroxyl groups. This result indicates that the remaining methoxy group was not in a position to interfere with metal chelation. Additional support for the position of the remaining methoxy group is found in the work of Feutrill and Mirrington. ${ }^{5}$ They treated a series of anisole compounds with sodium ethanethiolate and found 
that ortho-methoxy groups underwent complete demethylation while para-methoxy groups yielded selective mono-demethylation.

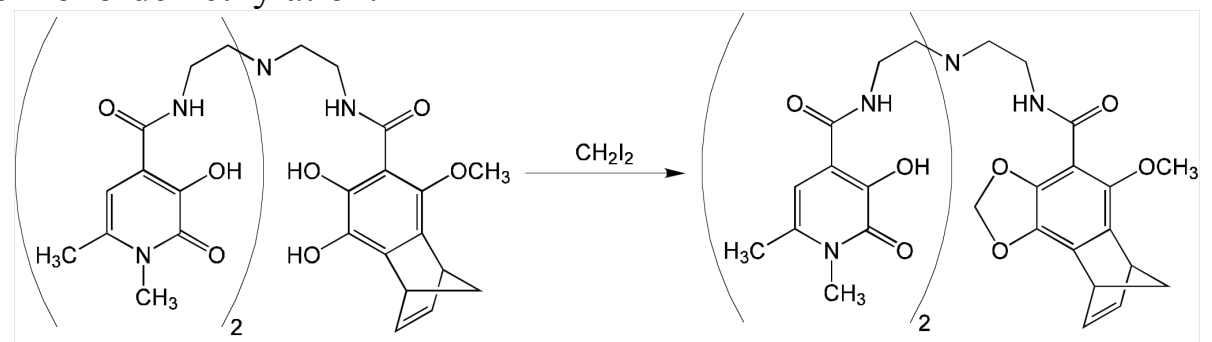

The intermediate was dissolved in water in a concentration between 0.1 and $6.5 \mathrm{mM}$, and five drops of DMSO were added. One equivalent of $\mathrm{GdCl}_{3} \cdot 6 \mathrm{H}_{2} \mathrm{O}$ per chelating group was then added, and the $\mathrm{pH}$ of the resulting solution was adjusted to 7 using $0.1 \mathrm{~N} \mathrm{NaOH}$ and $0.1 \mathrm{~N} \mathrm{HCl}$ solutions. The reaction mixture was heated to $80{ }^{\circ} \mathrm{C}$ for $1 \mathrm{~h}$, at which point the $\mathrm{pH}$ was readjusted to 7 . The reaction mixture was allowed to sit at ambient temperate for $15 \mathrm{~h}$. The $\mathrm{pH}$ was brought to 10 using a $0.1 \mathrm{~N} \mathrm{NaOH}$ solution to precipitate any unchelated gadolinium as $\mathrm{Gd}(\mathrm{OH})_{3}$. $\mathrm{Gd}(\mathrm{OH})_{3}$ was removed by filtration through a $0.45 \mu \mathrm{m}$ syringe filter. The $\mathrm{pH}$ of the filtered solution was brought to 7 using a $0.1 \mathrm{~N} \mathrm{HCl}$ solution, and the resulting solution was directly used for $T_{1}$ acquisition. After measurement of $T_{1}$, solutions were analyzed for $\mathrm{Gd}$ concentration, and the $T_{1}$ and Gd concentration data were used to calculate per Gd relaxivity. Per Gd relaxivity was multiplied by the number of chelates per polymer (as determined by NMR spectroscopy of polymers 9) to determine molecular relaxivity values.

\section{Intermediate of methyl ether removal from 12:}<smiles>COc1c(C(=O)NCCN2CCCCC2)c(O)c(O)c2c1C1C=CC2C1</smiles>

ESI-MS calcd for $\mathrm{C}_{35} \mathrm{H}_{42} \mathrm{~N}_{6} \mathrm{O}_{10}[\mathrm{M}+\mathrm{H}]^{+}$: 707.3, found 707.4.

\section{Complex 11:}

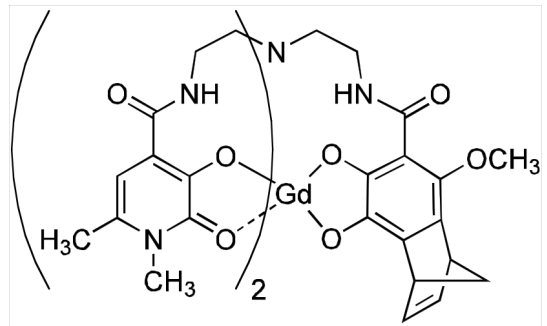

ESI-MS calcd for $\mathrm{C}_{35} \mathrm{H}_{38} \mathrm{GdN}_{6} \mathrm{O}_{10}\left[\mathrm{M}-\mathrm{CH}_{3}+\mathrm{Na}+2 \mathrm{H}\right]^{+}$: Gd isotope pattern centered at 870.2, found $\mathrm{Gd}$ isotope pattern centered at 870.1. Maximum Gd concentrations ranged from $0.0054 \%$ to $0.0176 \%$. Relaxivity $\left(r_{1}\right): 10.5 \pm 0.8 \mathrm{mM}^{-1} \mathrm{~s}^{-1}$ (error is given as the standard deviation). 


\section{Polymer 10a:}

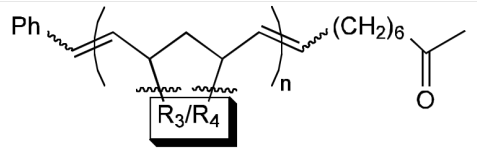

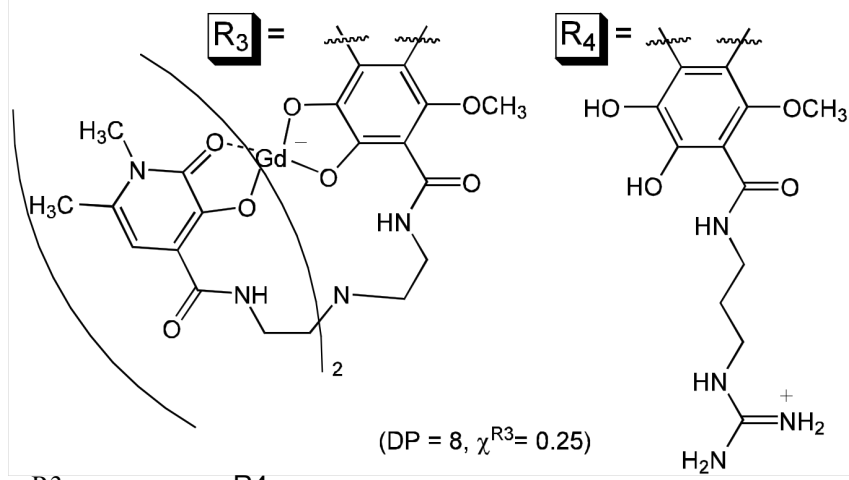

$\chi^{\mathrm{R} 3}=0.25, \chi^{\mathrm{R} 4}=0.75$. Maximum Gd concentrations ranged from $0.0040 \%$ to $0.0046 \%$. Ionic relaxivity $\left(r_{1}\right): 10.1 \pm 0.5 \mathrm{mM}^{-1} \mathrm{~s}^{-1}$; Molecular relaxivity $\left(r_{1}\right)$ : $18.8 \pm 0.9 \mathrm{mM}^{-1} \mathrm{~s}^{-1}$ (errors are given as standard deviations).

Polymer 10b:
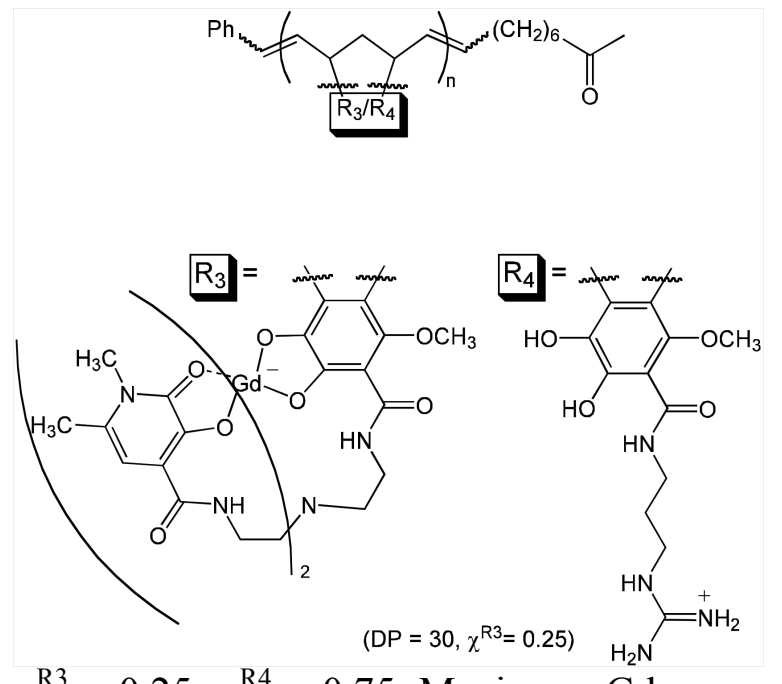

$\chi^{\mathrm{R} 3}=0.25, \chi^{\mathrm{R} 4}=0.75$. Maximum $\mathrm{Gd}$ concentrations ranged from $0.0005 \%$ to $0.0048 \%$.

Ionic relaxivity $\left(r_{1}\right): 14.8 \pm 0.2 \mathrm{mM}^{-1} \mathrm{~s}^{-1}$; Molecular relaxivity $\left(r_{1}\right): 111.0 \pm 1.5 \mathrm{mM}^{-1} \mathrm{~s}^{-1}$ (errors are given as standard deviations).

\section{$\mathbf{N}$-(3-aminopropyl)guanidine bis-trifluoroacetic acid salt: \\ $+\mathrm{H}_{3} \mathrm{~N}$<smiles>COC(=O)C(F)(F)F</smiles>

$N$-(bis-Boc-guanyl)-N'-Boc-1,3-diaminopropane (1.00 g, $2.40 \mathrm{mmol}$ ) was dissolved in 20 $\mathrm{mL}$ of $95 \%$ trifluoroacetic acid (TFA), $2.5 \%$ water, and $2.5 \%$ triisopropylsilane (v/v/v). After 14 $\mathrm{h}$, the volume was reduced to a sticky residue under a stream of air. The residue was washed 
with diethyl ether, dissolved in water, and freeze dried to yield $0.568 \mathrm{~g}(69 \%)$ of the desired salt as an extremely viscous, colorless oil. ${ }^{1} \mathrm{H}$ NMR $\left(300 \mathrm{MHz}, \mathrm{D}_{2} \mathrm{O}\right): \delta=1.96-2.06\left(\mathrm{~m}, 2 \mathrm{H}, \mathrm{CH}_{2}-\right.$ $\left.\mathrm{CH}_{2}-\mathrm{CH}_{2}\right), 3.11\left(\mathrm{t}, 2 \mathrm{H}, \mathrm{J}=8.0 \mathrm{~Hz}, \mathrm{~N}-\mathrm{CH}_{2}-\mathrm{CH}_{2}\right), 3.35\left(\mathrm{t}, 2 \mathrm{H}, \mathrm{J}=7.0 \mathrm{~Hz}, \mathrm{~N}-\mathrm{CH}_{2}-\mathrm{CH}_{2}\right)$; ${ }^{13} \mathrm{C} \mathrm{NMR}$ $\left(75 \mathrm{MHz}, \mathrm{D}_{2} \mathrm{O}\right): \delta=27.6\left(\mathrm{CH}_{2}-\mathrm{CH}_{2}-\mathrm{CH}_{2}\right), 38.3\left(\mathrm{~N}-\mathrm{CH}_{2}\right), 39.7\left(\mathrm{~N}-\mathrm{CH}_{2}\right), 158.3\left(\mathrm{C}-(\mathrm{N})_{3}\right)$; ESIMS calcd for $\mathrm{C}_{4} \mathrm{H}_{12} \mathrm{~N}_{4}[2 \mathrm{M}+\mathrm{H}]^{+}$: 233.3, found 233.1; Anal. Calcd for $\mathrm{C}_{8} \mathrm{H}_{14} \mathrm{~F}_{6} \mathrm{~N}_{4} \mathrm{O}_{4}$ : C, 27.91; H, 4.10; F, 33.12; N, 16.28. Found: C, 27.87; H, 4.24; F, 31.17; N, 15.55.

\section{$N$-(bis-Boc-guanyl)-N'-Boc-1,3-diaminopropane:}

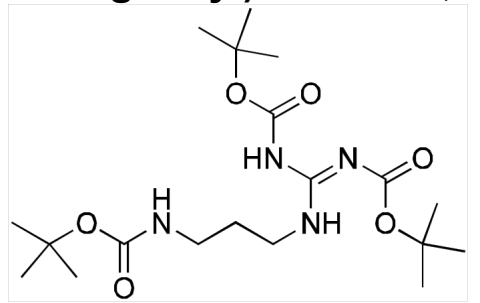

To a solution of $N$-Boc-1,3-diaminopropane $(5.00 \mathrm{~mL}, 28.6 \mathrm{mmol})$ in dimethylformamide (DMF) (100 mL) was added diisopropylethylamine (DIEA) $(3.72 \mathrm{~mL}, 21.4 \mathrm{mmol})$ and bis-Bocguanylpyrazole $(3.31 \mathrm{~g}, 10.7 \mathrm{mmol})$. The reaction mixture was stirred for $14 \mathrm{~h}$, at which time DMF was removed under reduced pressure. Water $(20 \mathrm{~mL})$ was added, and the mixture was extracted with dichloromethane. The organic layer was dried over magnesium sulfate, and solvent was removed under reduced pressure. Purification was performed using silica gel chromatography $(5: 1 \rightarrow 2: 1$ hexanes/ethyl acetate) to yield $3.07 \mathrm{~g}(69 \%)$ of the desired product as a white solid. ${ }^{1} \mathrm{H}$ NMR $\left(300 \mathrm{MHz}, \mathrm{CDCl}_{3}\right): \delta=1.43\left(\mathrm{~s}, 9 \mathrm{H}, \mathrm{CH}_{3}\right), 1.49\left(\mathrm{~s}, 18 \mathrm{H}, \mathrm{CH}_{3}\right), 1.64-$ $1.72\left(\mathrm{~m}, 2 \mathrm{H}, \mathrm{CH}_{2}-\mathrm{CH}_{2}-\mathrm{CH}_{2}\right), 3.10-3.17\left(\mathrm{~m}, 2 \mathrm{H}, \mathrm{NH}-\mathrm{CH}_{2}-\mathrm{CH}_{2}\right), 3.44-3.50\left(\mathrm{~m}, 2 \mathrm{H}, \mathrm{NH}-\mathrm{CH}_{2}-\right.$ $\left.\mathrm{CH}_{2}\right), 5.60(\mathrm{~s}, 1 \mathrm{H}, \mathrm{NH}), 8.34(\mathrm{t}, 1 \mathrm{H}, J=5.6 \mathrm{~Hz}, \mathrm{NH}), 11.41(\mathrm{~s}, 1 \mathrm{H}, \mathrm{N} H) ;{ }^{13} \mathrm{C} \mathrm{NMR}(75 \mathrm{MHz}$, $\left.\mathrm{CDCl}_{3}\right): \quad \delta=28.3\left(\mathrm{CH}_{3}\right), 28.5\left(\mathrm{CH}_{3}\right), 28.7\left(\mathrm{CH}_{3}\right), 30.4\left(\mathrm{CH}_{2}-\mathrm{CH}_{2}-\mathrm{CH}_{2}\right), 37.3\left(\mathrm{NH}-\mathrm{CH}_{2}-\mathrm{CH}_{2}\right)$, $37.8\left(\mathrm{NH}-\mathrm{CH}_{2}-\mathrm{CH}_{2}\right), 79.0\left(\mathrm{C}\left(\mathrm{CH}_{3}\right)_{3}\right), 79.4\left(\mathrm{C}\left(\mathrm{CH}_{3}\right)_{3}\right), 83.4\left(C\left(\mathrm{CH}_{3}\right)_{3}\right), 153.4,156.4,156.9$, 163.5; TLC: $\mathrm{R}_{\mathrm{f}}=0.36$ (2:1 hexanes/ethyl acetate); ESI-MS calcd for $\mathrm{C}_{19} \mathrm{H}_{36} \mathrm{~N}_{4} \mathrm{O}_{6}[\mathrm{M}+\mathrm{H}]^{+}$: 417.2713, found 417.2693.

\section{Estimation of Rotational Correlation Time}

Values of the rotational correlation time $\left(\tau_{R}\right)$ were estimated using the Debye-Stokes equation shown below and data acquired for other linear $\mathrm{Gd}^{\mathrm{III}}$ containing polymers. ${ }^{6,7}$ Assuming that the microviscosity and density of the polymers are the same, the ratio of molecular radii can be expressed by the ratio of the molecular weights. While the Debye-Stokes equation provides estimation for spherical molecules, the ROMP-derived polymers and polymers in references 6 and 7 are both linear and the ratio of their molecular weights is used only as an approximation.

$$
\tau_{R}=\frac{4 \pi \eta r_{e f f}^{3}}{3 k_{B} T}
$$

Debye-Stokes equation ( $\eta=$ microviscosity, $r_{\text {eff }}=$ molecular radius, $k_{B}=$ the Boltzmann constant, $T=$ temperature) 
weight $(\mathrm{MW}), \tau_{R}$, and per Gd relaxivity for two linear polymers from the literature ( $\tau_{g}$, global motion correlation time, was used to estimate $\tau_{R}$ for comparison to ROMP-derived polymers 10a and $\mathbf{1 0 b}$ because of the rigid connection to the polymer backbone). The $\tau_{R}$ data for ROMPderived polymers 10a and 10b were estimated by plotting $\tau_{R}$ vs. MW for the published polymers, and using the resulting slope with the molecular weights of polymers $10 a$ and $10 \mathbf{b}$. The ratio of $\tau_{R}$ to relaxivity was then examined, and the relaxivity values in parentheses for 10a and 10b would be expected for linear polymers with their molecular weights and estimated $\tau_{R}$ values. These estimates match up very well with the actual measurements indicating that there is relaxivity increase due to increase in $\tau_{R}$. Thus, the observed increase is proportional to what is seen in linear polymers of $\mathrm{Gd}^{\mathrm{III}}$ diethylenetriaminepentaacetic acid (DTPA).

\begin{tabular}{|c|c|c|c|}
\hline Polymer & MW (Da) & $\begin{array}{c}\boldsymbol{\tau}_{\boldsymbol{R}}\left(\mathbf{1 0}^{\mathbf{- 1 2}}\right. \\
\mathbf{S})\end{array}$ & Per Gd relaxivity $\left(\mathbf{m M}^{\mathbf{- 1}} \mathbf{s}^{\mathbf{- 1}}\right)$ \\
\hline$\left[\mathrm{DTPA}-\mathrm{BA}\left(\mathrm{CH}_{2}\right)_{10}\right]_{\mathrm{x}}$ & 10300 & 2900 & 15.4 \\
\hline$\left[\mathrm{DTPA}-\mathrm{BA}\left(\mathrm{CH}_{2}\right)_{12}\right]_{\mathrm{x}}$ & 15700 & 4400 & 19.6 \\
\hline $\mathbf{1 0 a}$ & 4260 & 1200 & $10.1(10.7)$ \\
\hline $\mathbf{1 0 b}$ & 14200 & 4000 & $14.8(18.5)$ \\
\hline
\end{tabular}

\section{References}

(1) Doble, D. M. J.; Melchoir, M.; O’Sullivan, B.; Siering, C.; Xu, J.; Pierre, V. C.; Raymond, K. N. Inorg. Chem. 2003, 42, 4930-4937.

(2) Pontrello, J. K.; Allen, M. J.; Underbakke, E. S.; Kiessling, L. L. J. Am. Chem. Soc. 2005, 127, 14536-14537.

(3) Love, J. A.; Morgan, J. P. Trnka, T. M.; Grubbs, R. H. Angew. Chem., Int. Ed. 2002, 41, 4035-4037.

(4) Holmes, T. J.; Vennerstrom, V. J. J.; Choi, K. E. J. Org. Chem. 1984, 49, 4736-4738.

(5) Feutrill, G. I.; Mirrington, R. N. Tetrahedron Lett. 1970, 16, 1327-1328.

(6) Toth, E.; Helm, L.; Kellar, K. E.; Merbach, A. E. Chem. Eur. J. 1999, 5, 1202-1211.

(7) The Chemistry of Contrast Agents in Medical Magnetic Resonance Imaging; Merbach, A. E., Toth, E., Eds.; John Wiley \& Sons, Ltd.: New York, 2001. 


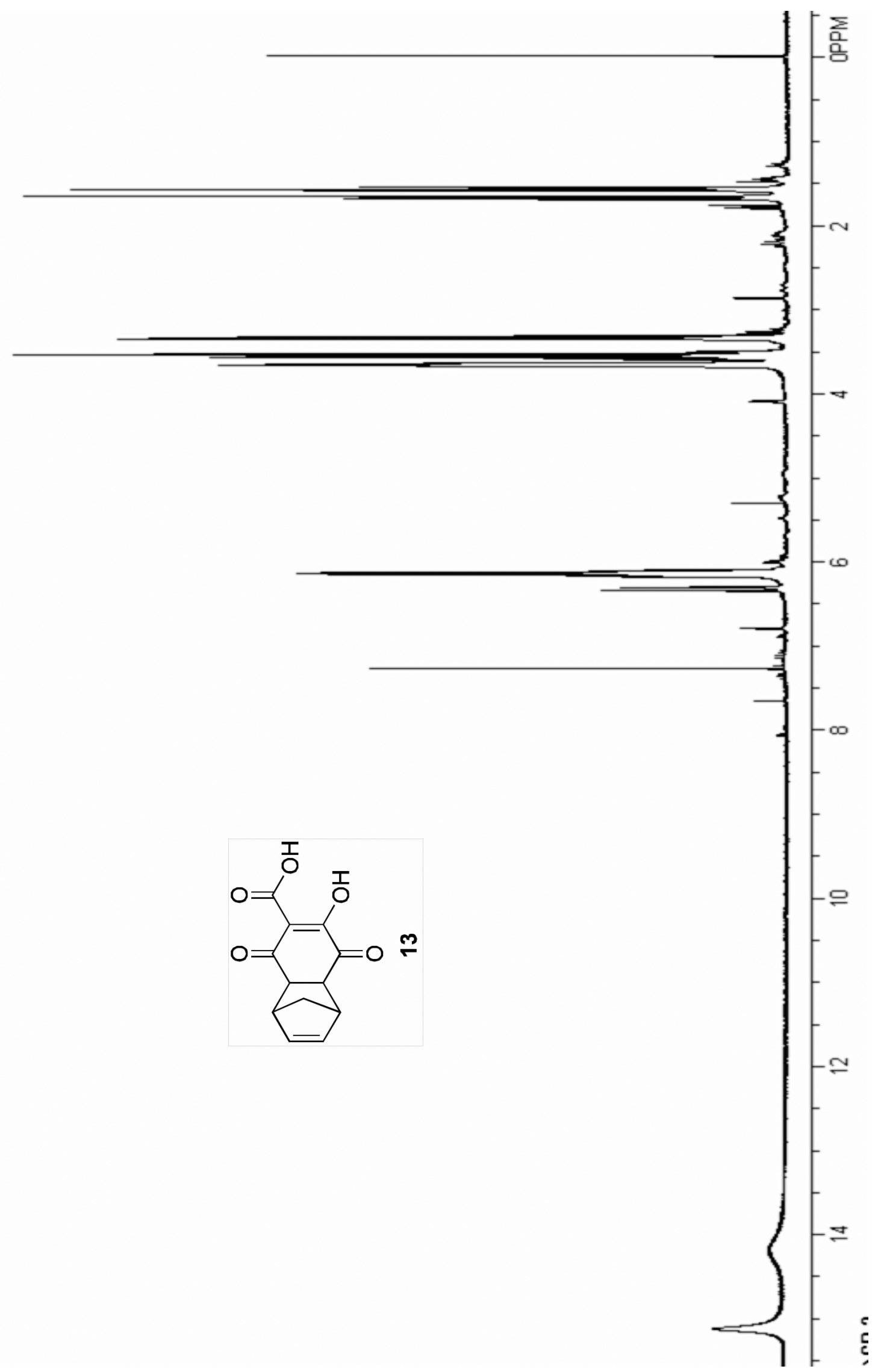




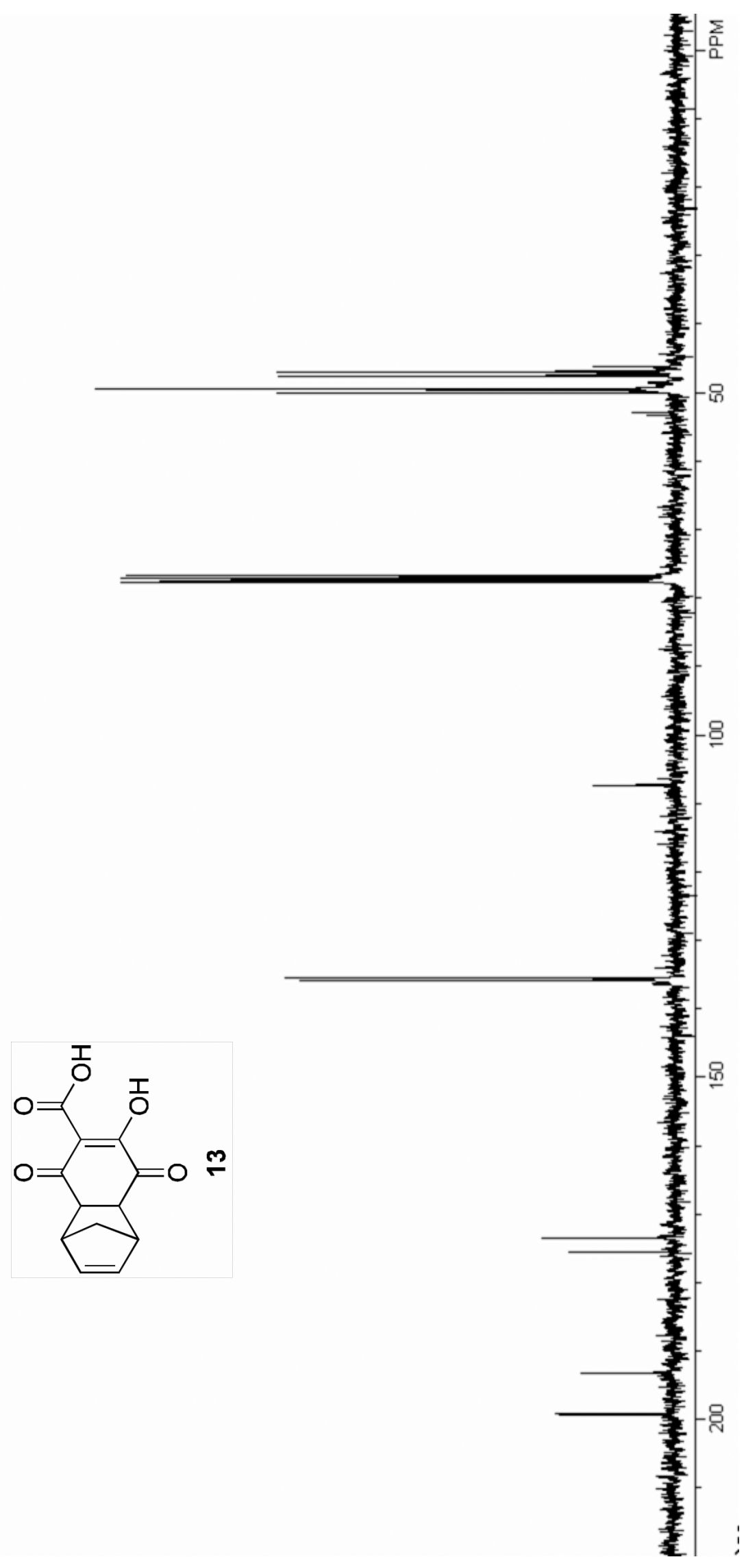




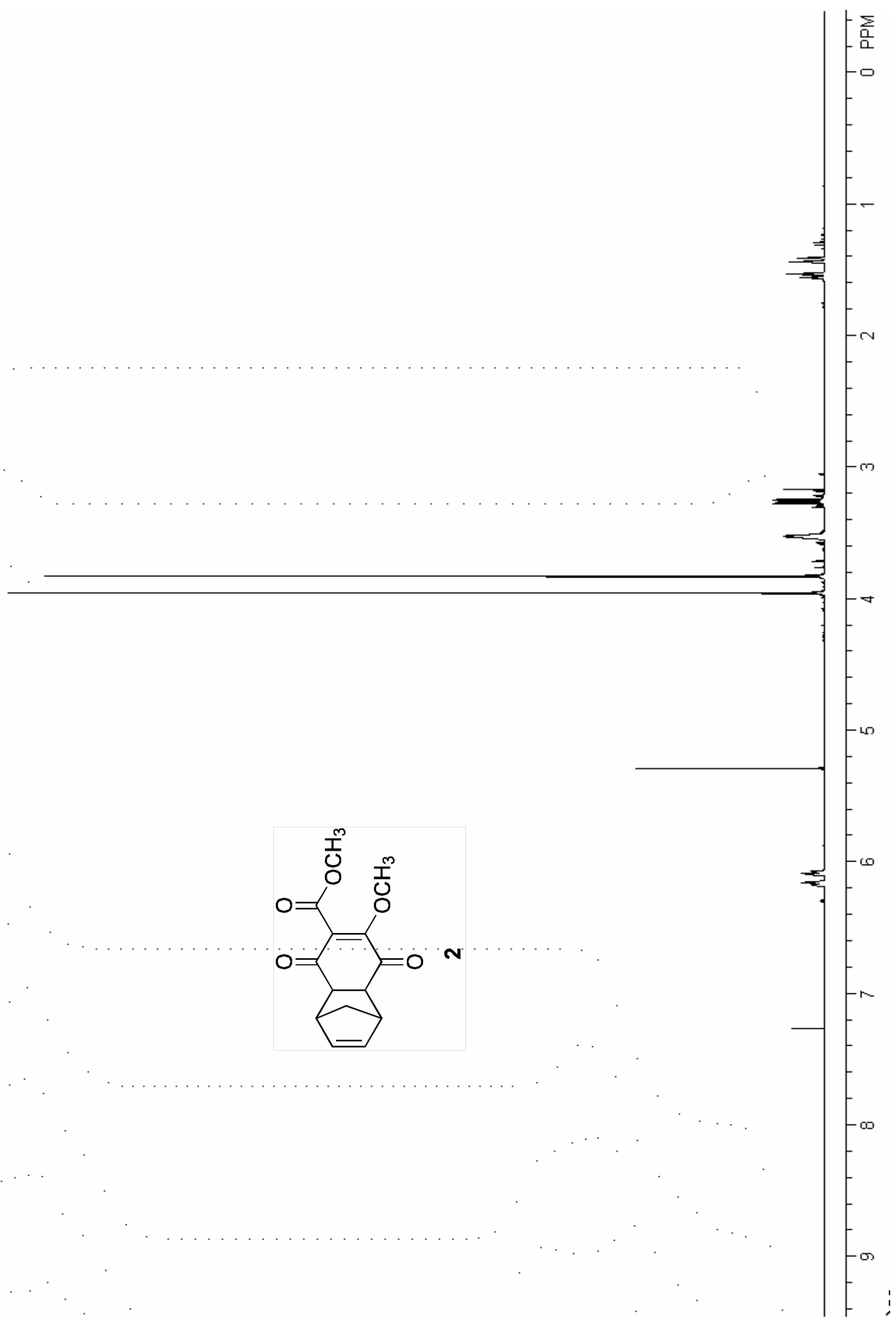




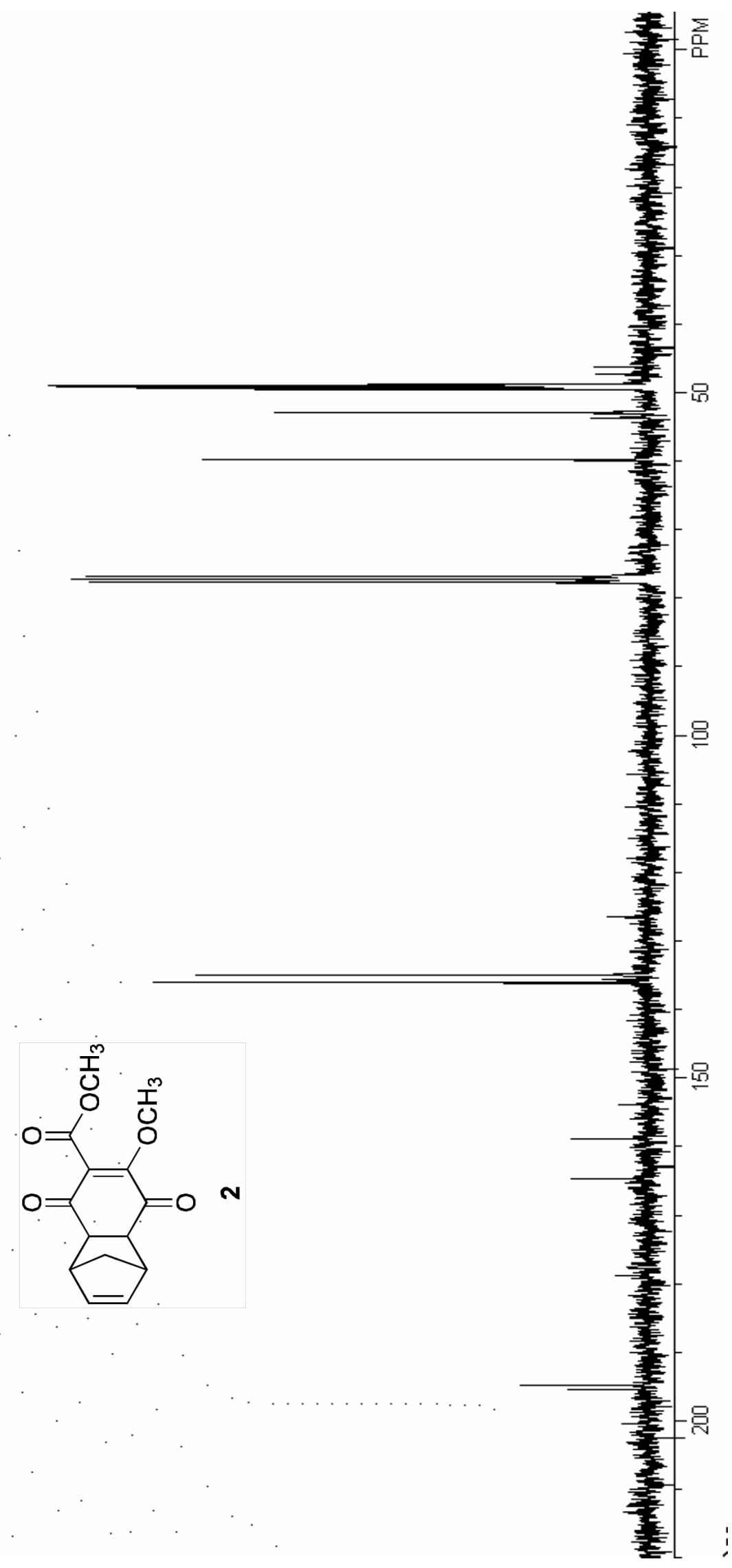




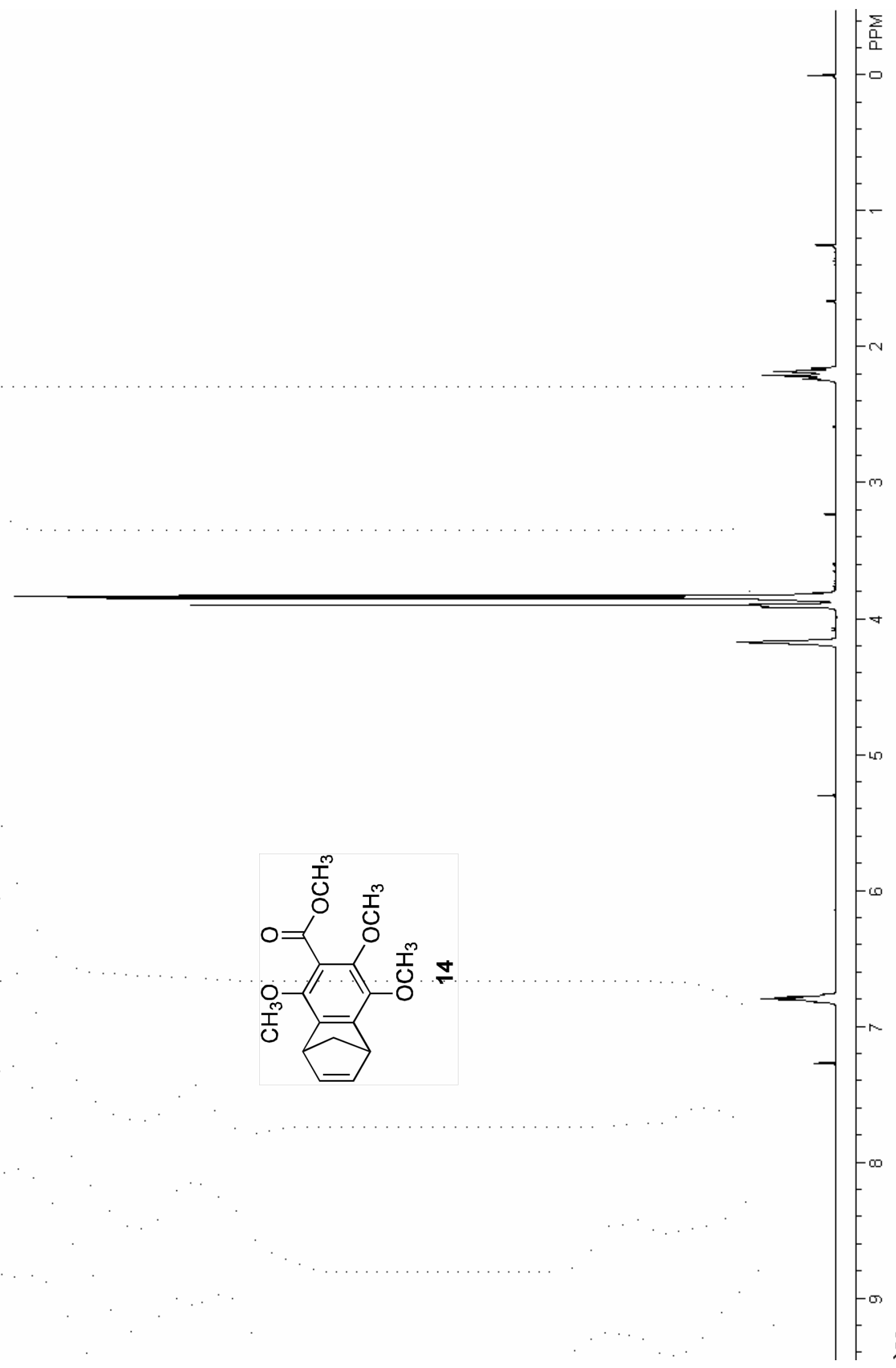









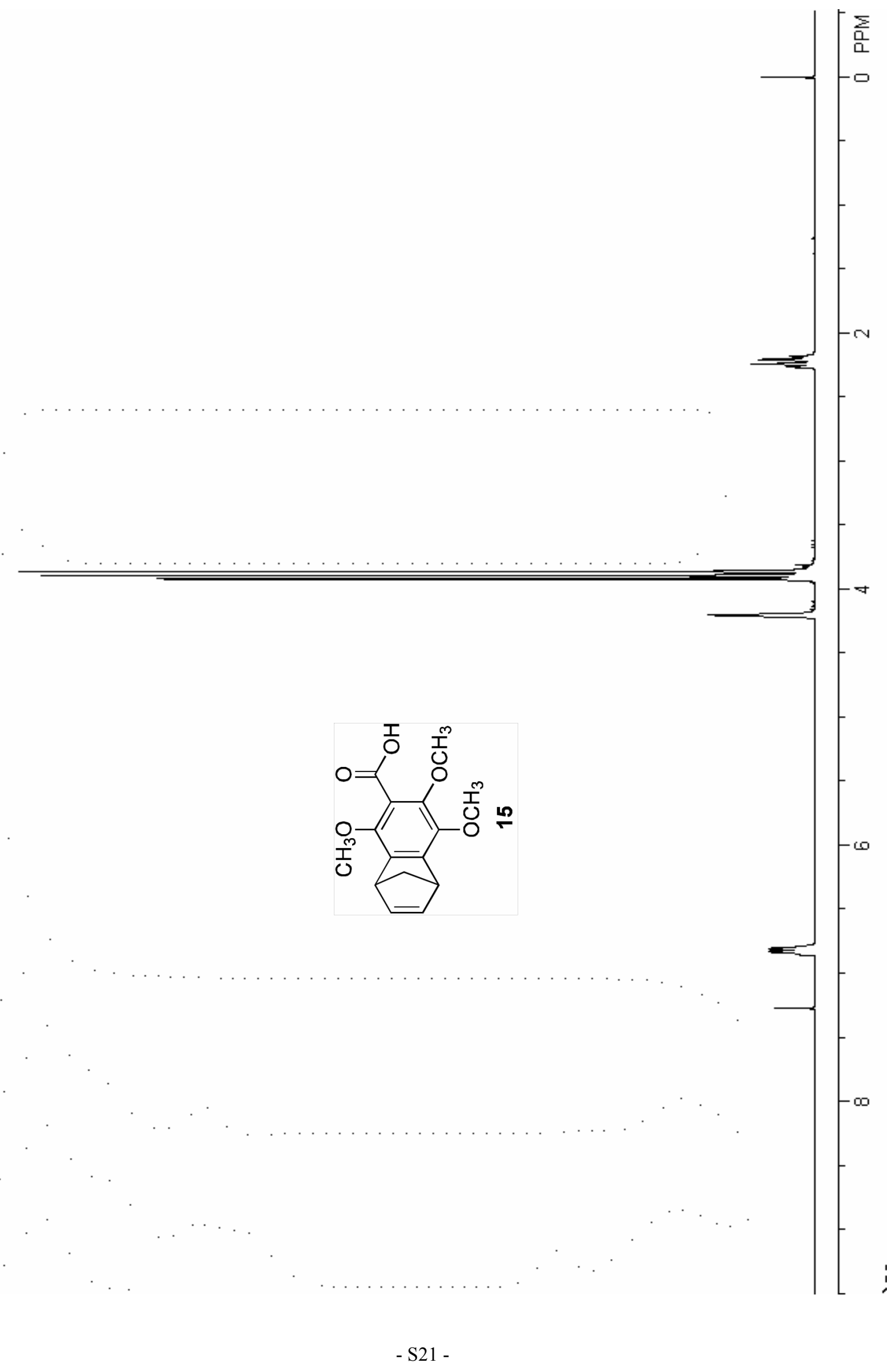




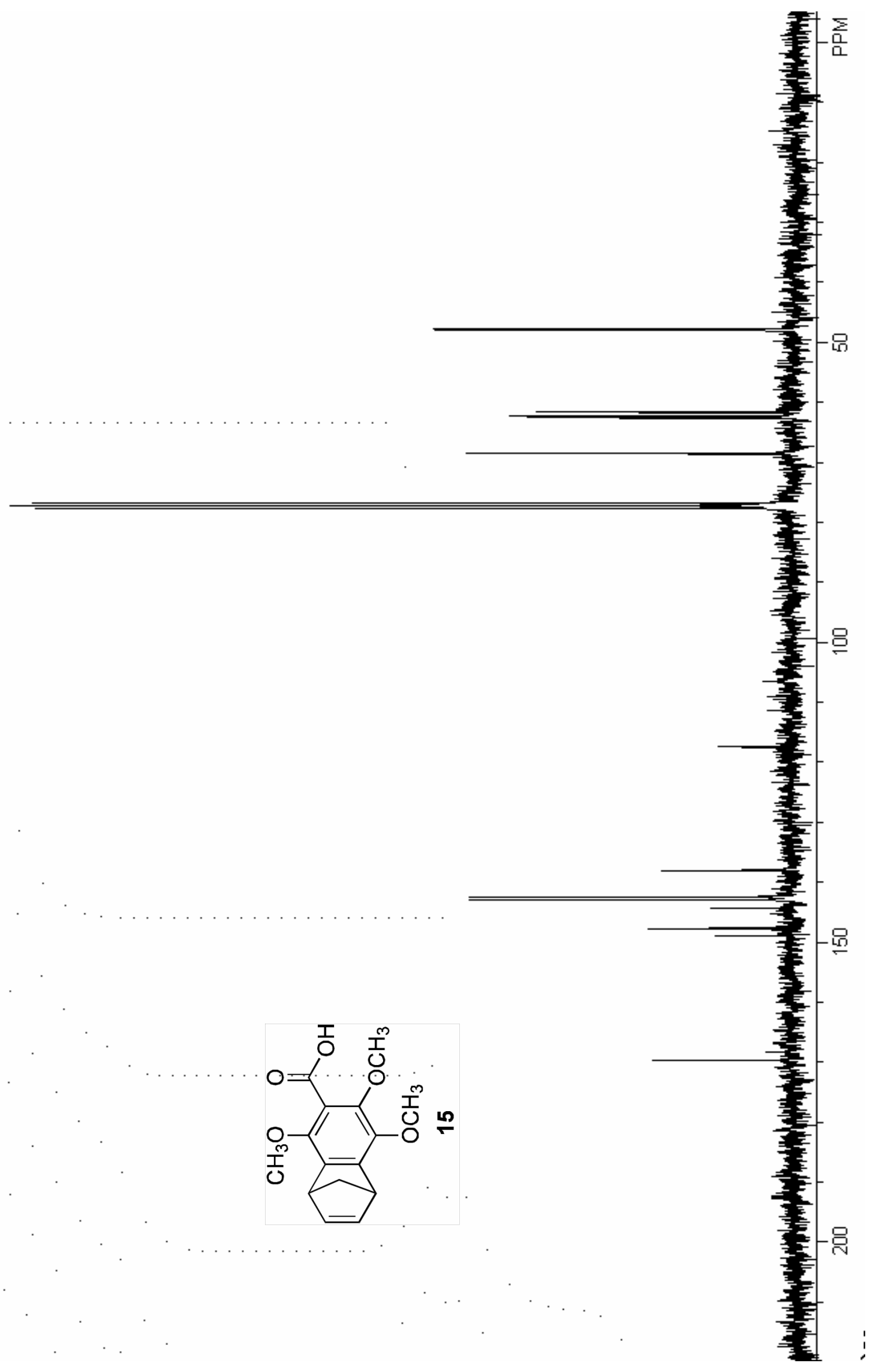









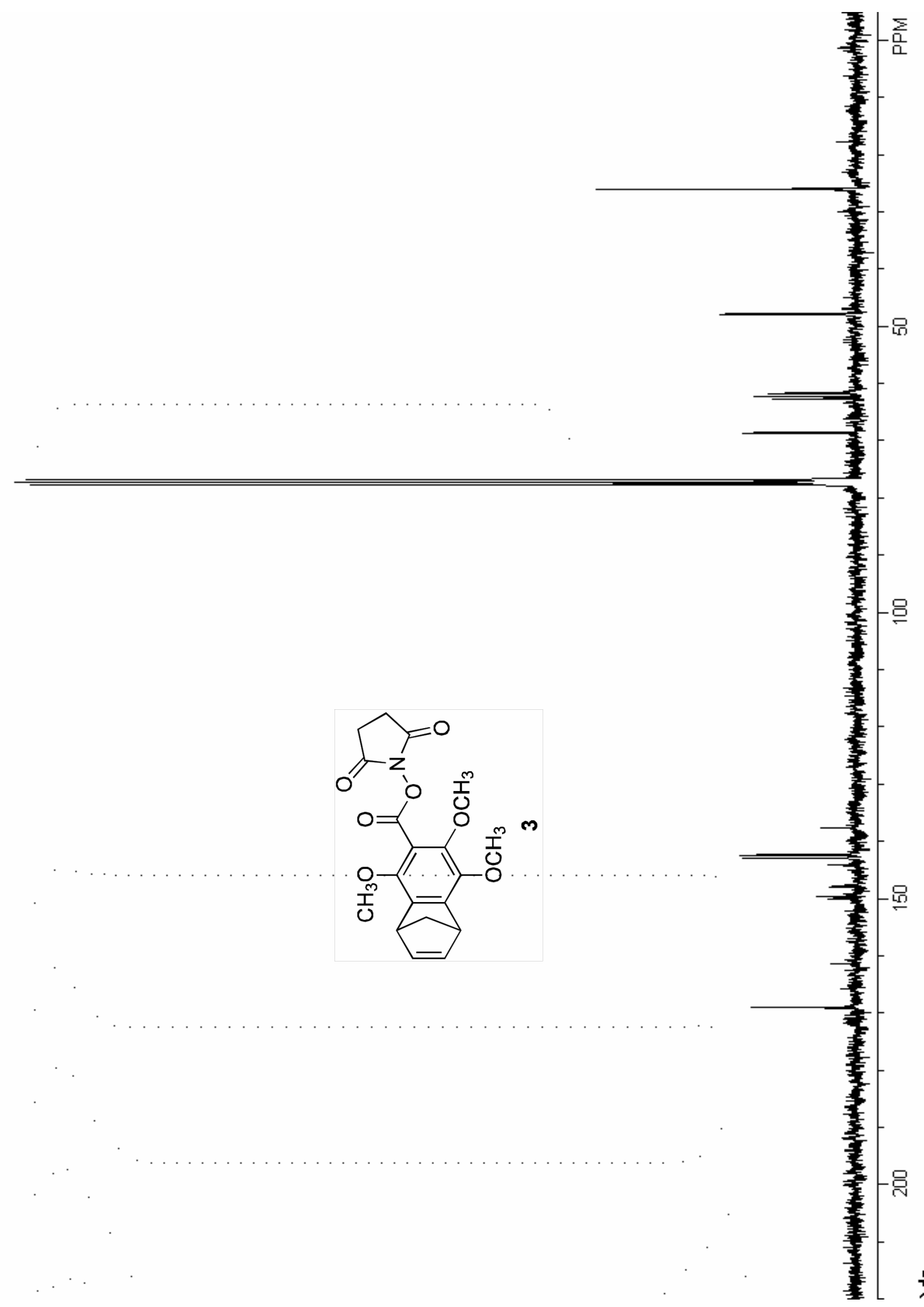




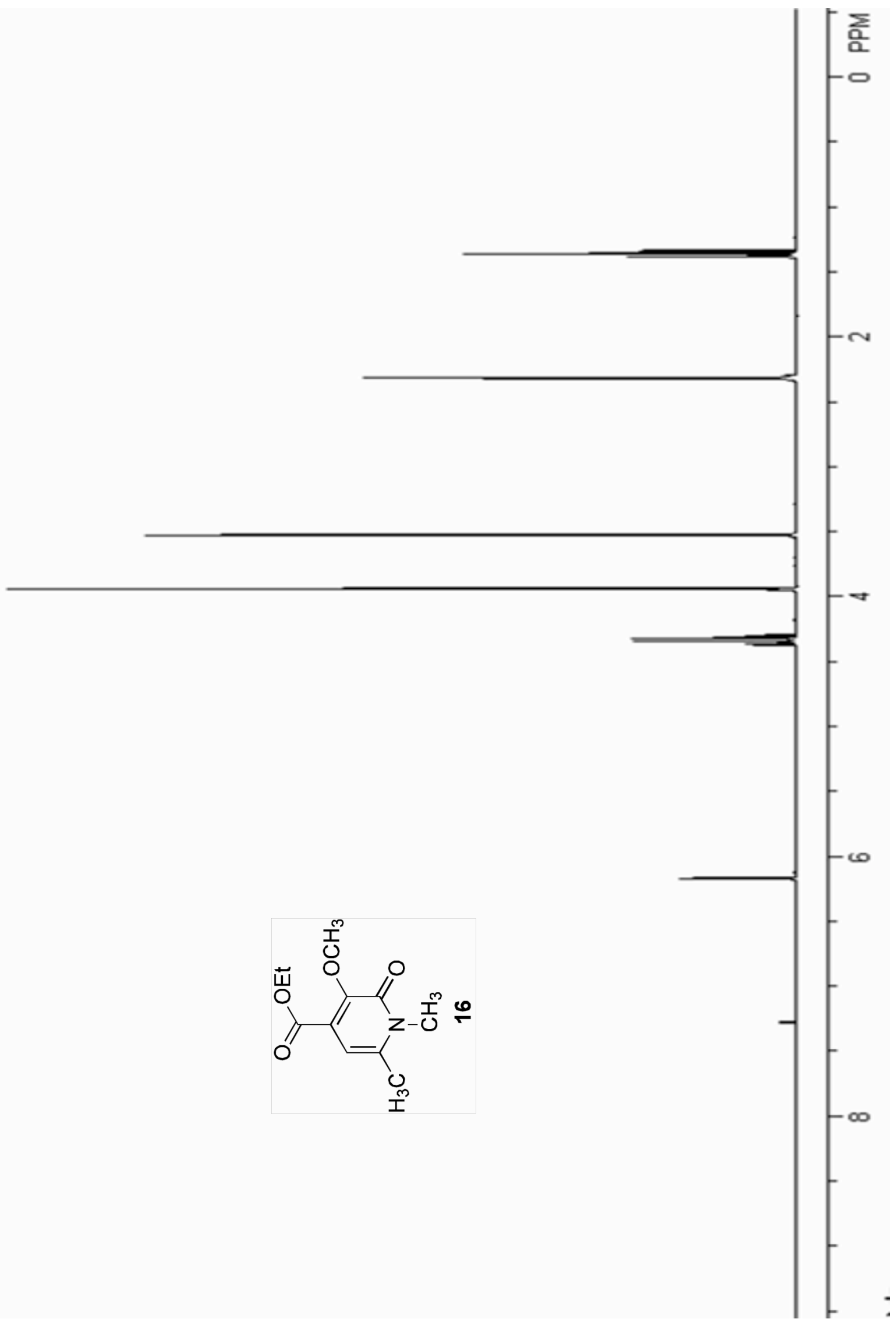














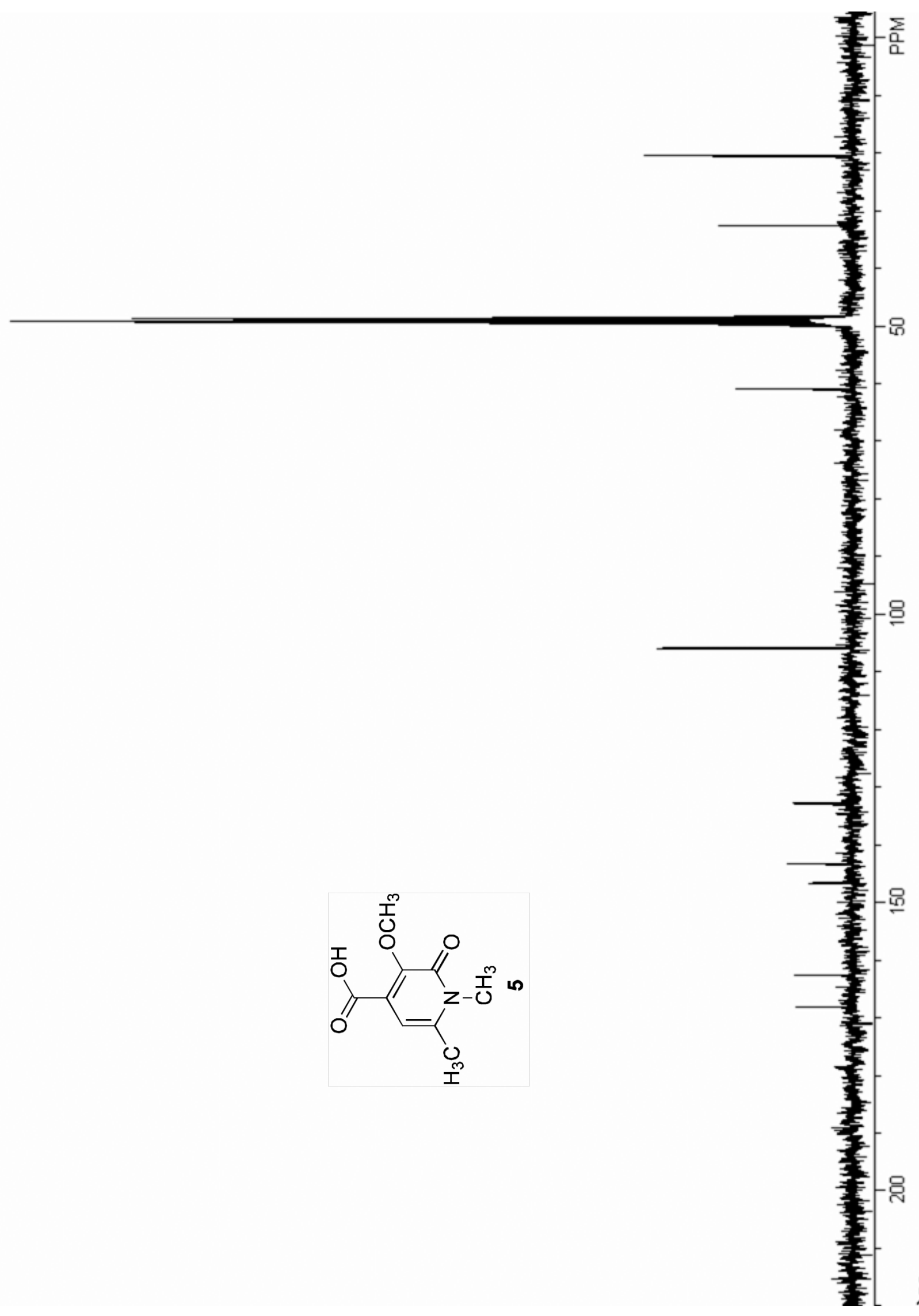




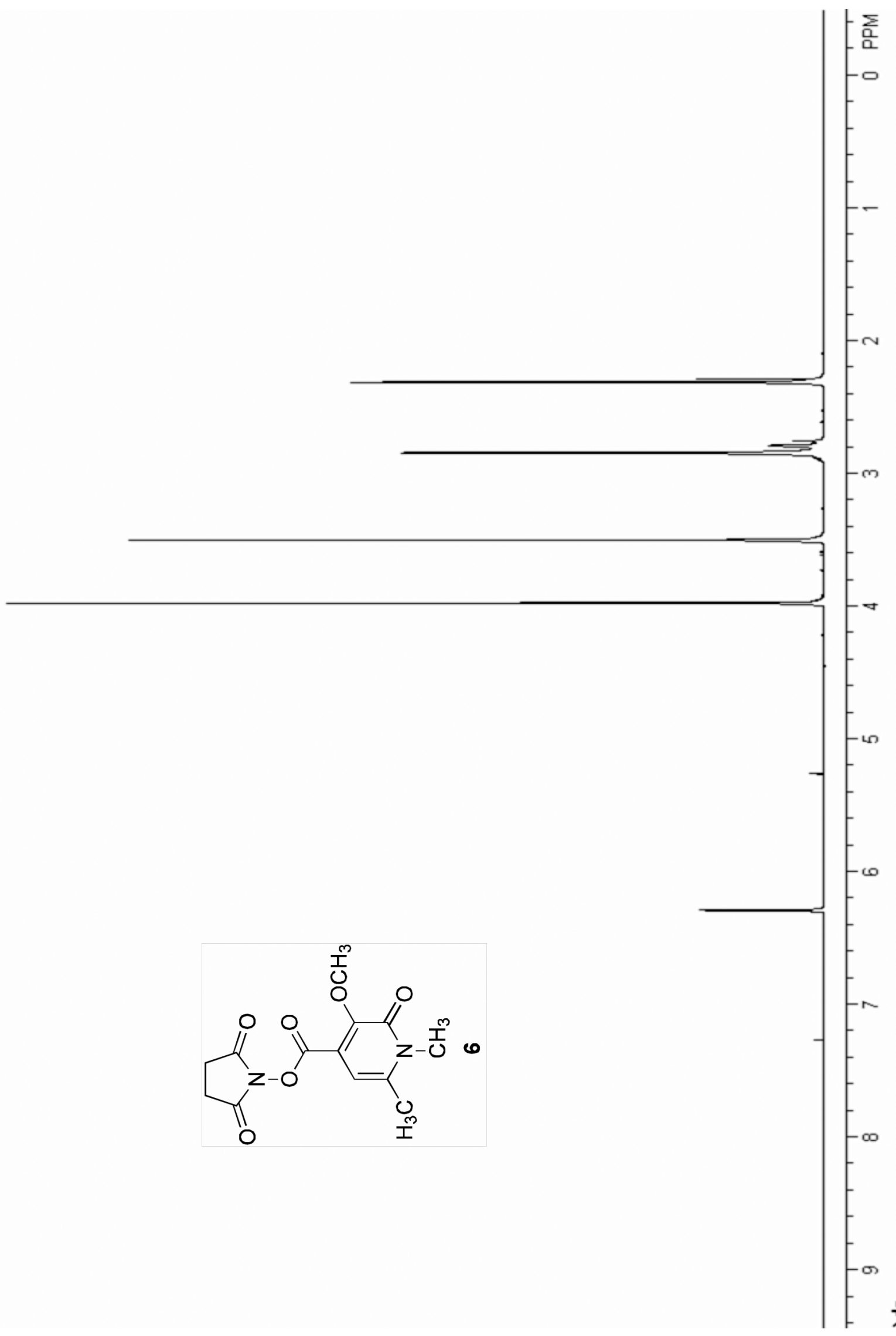




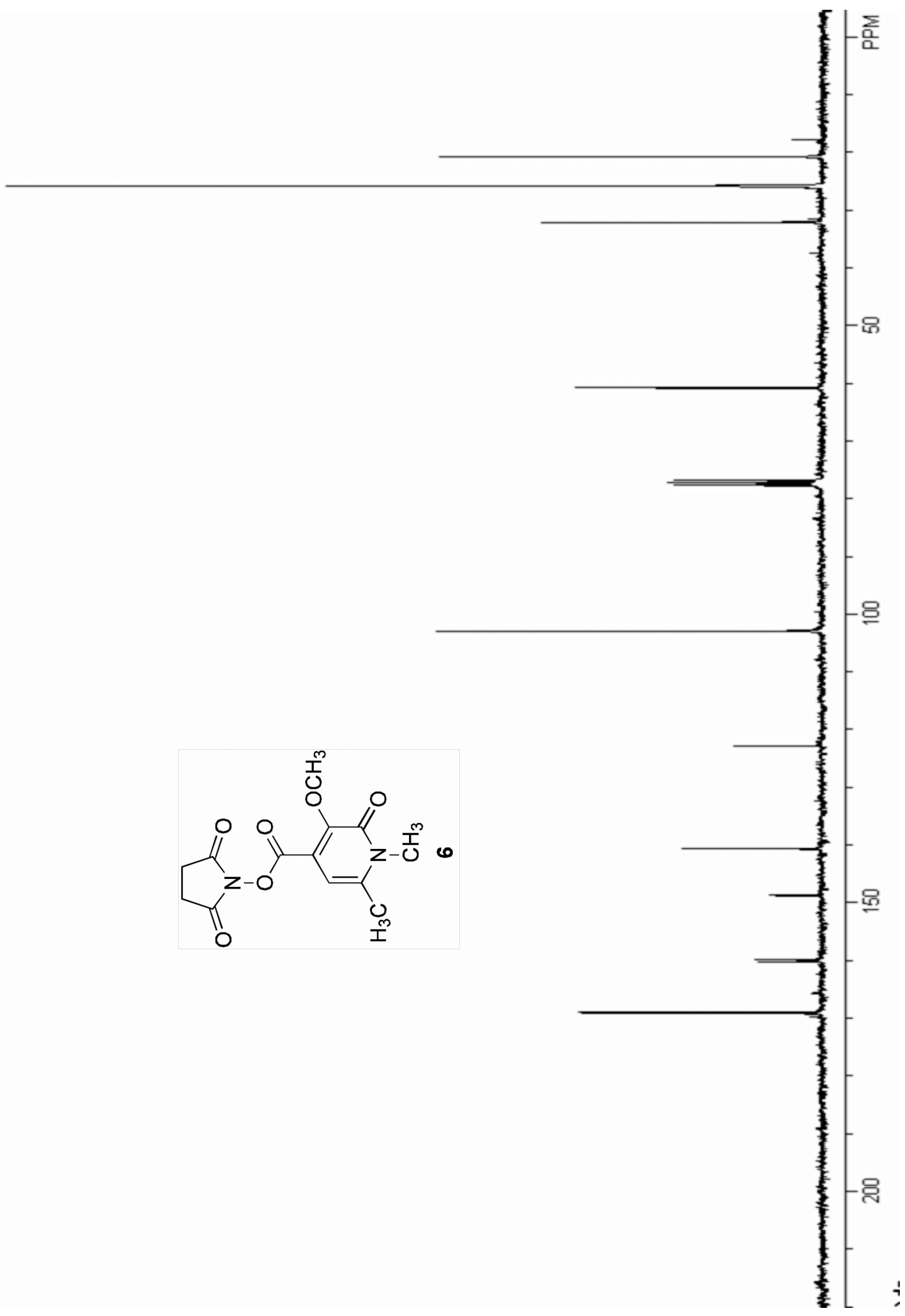









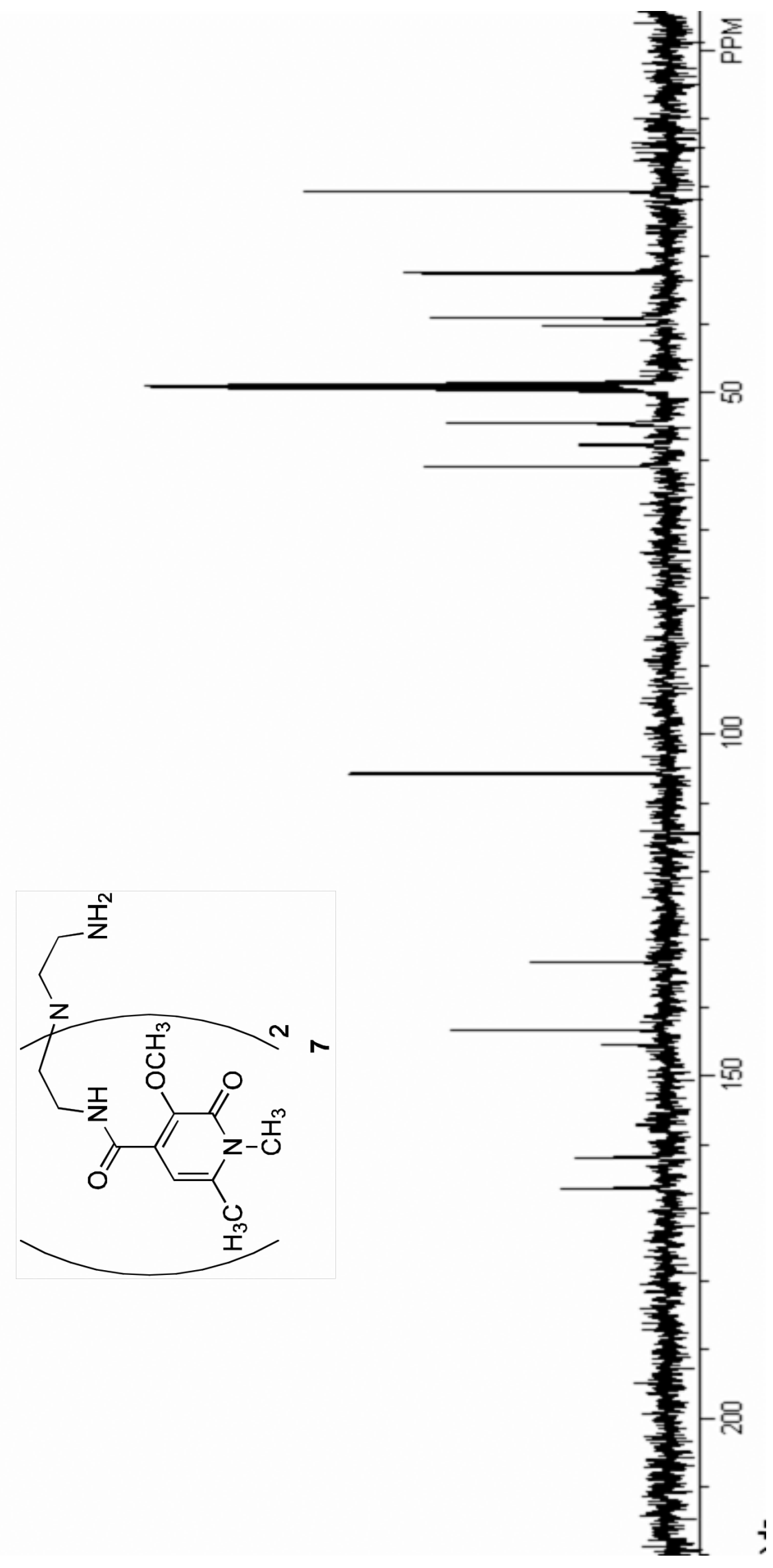




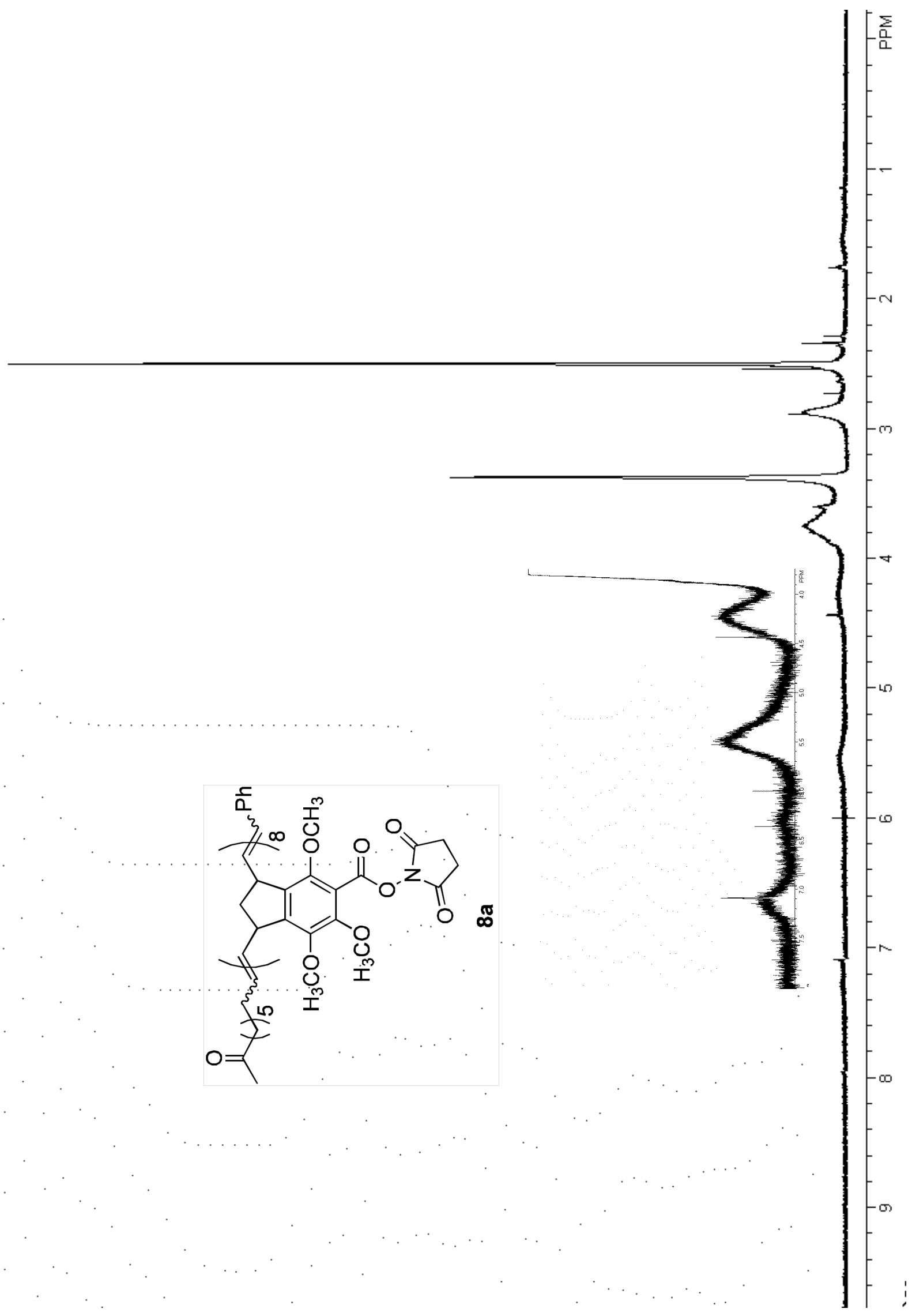




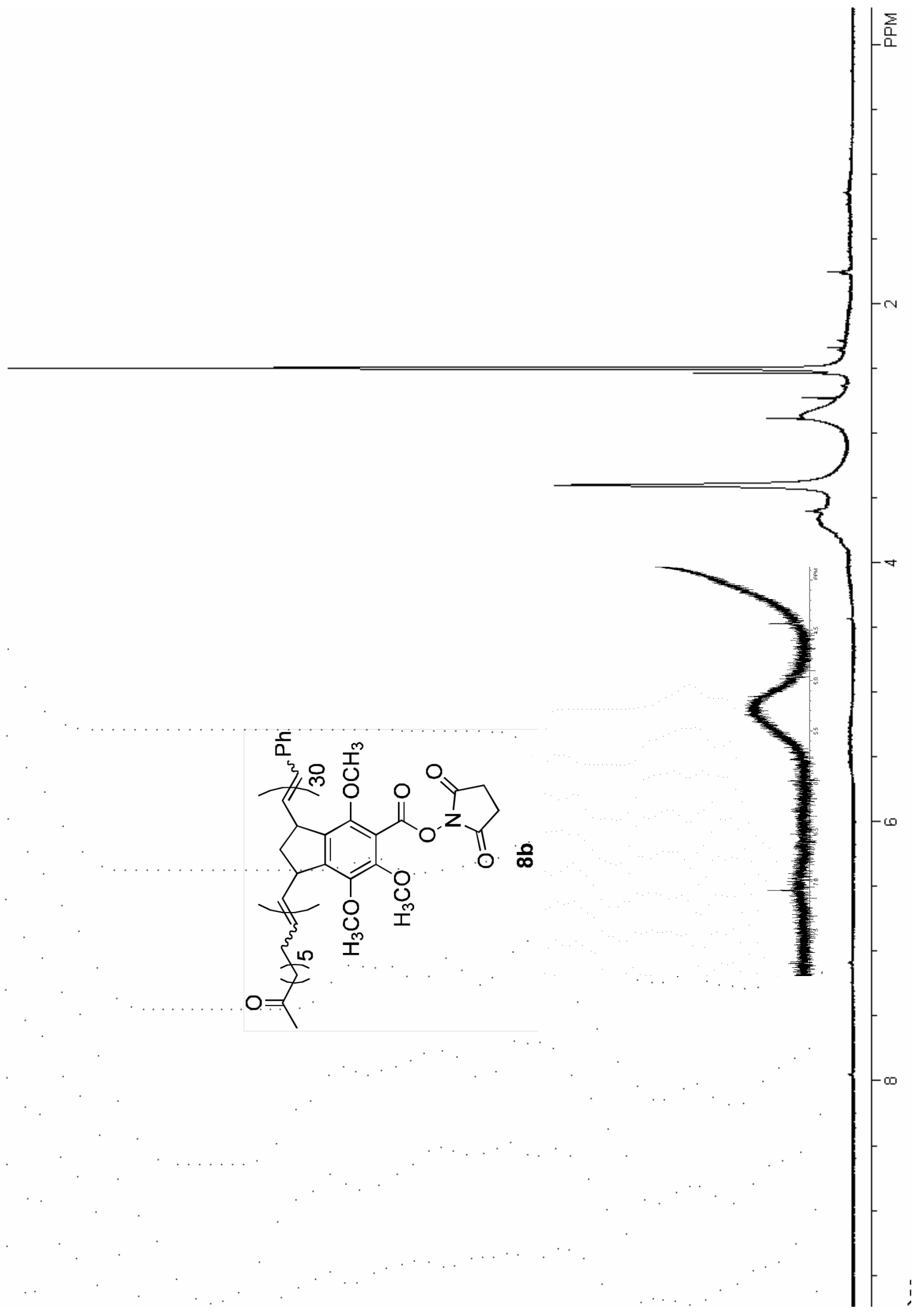




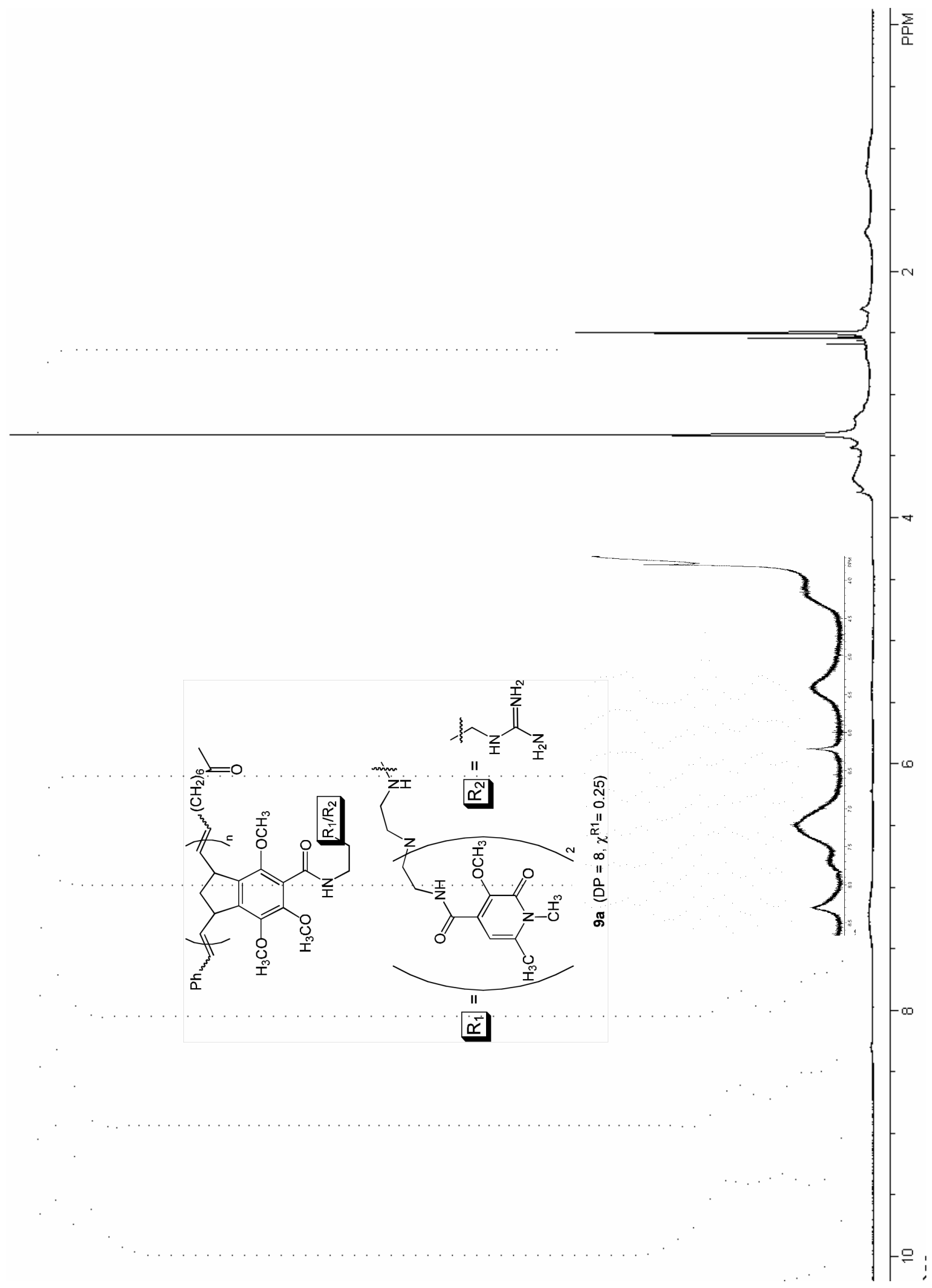




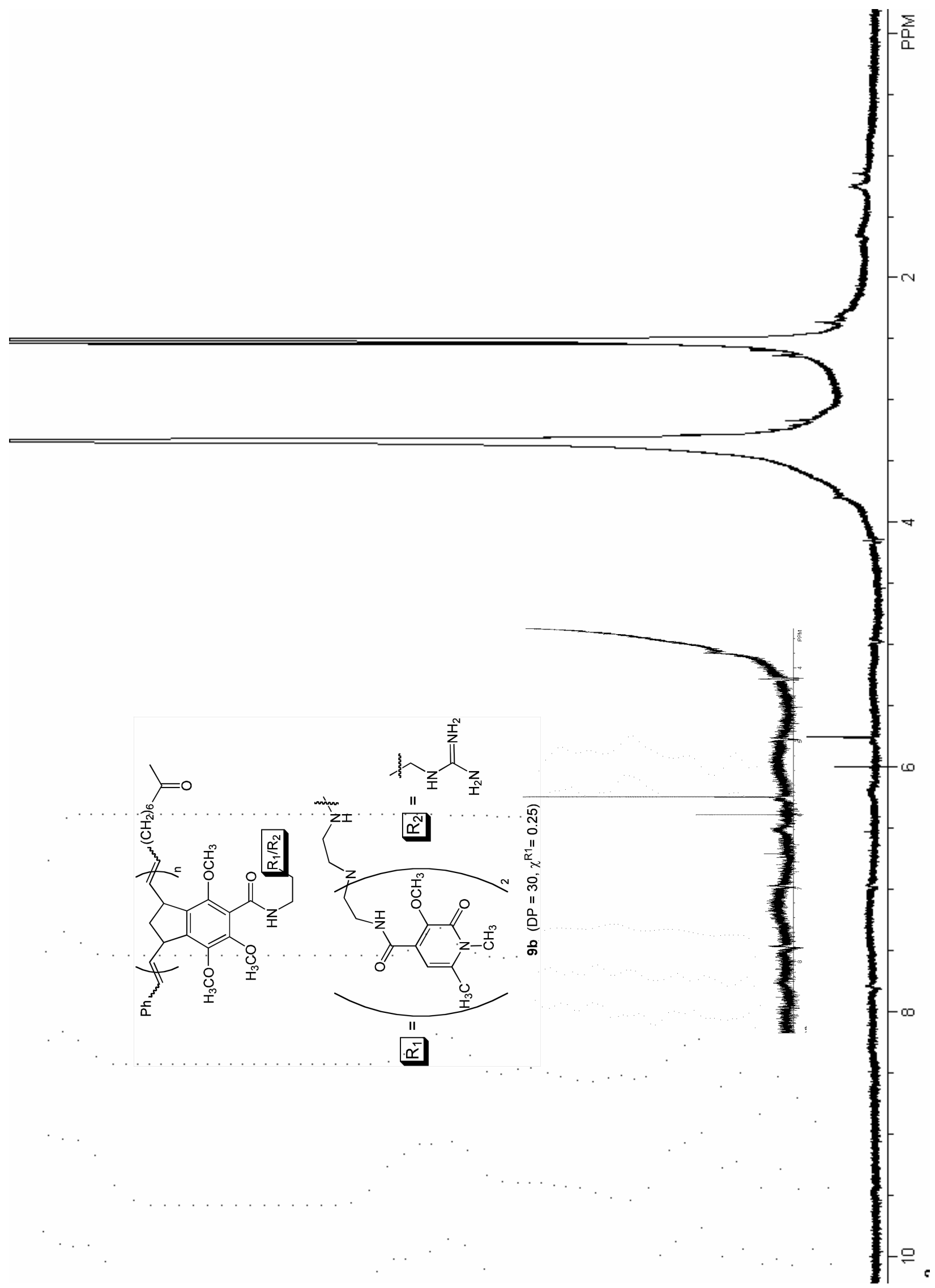




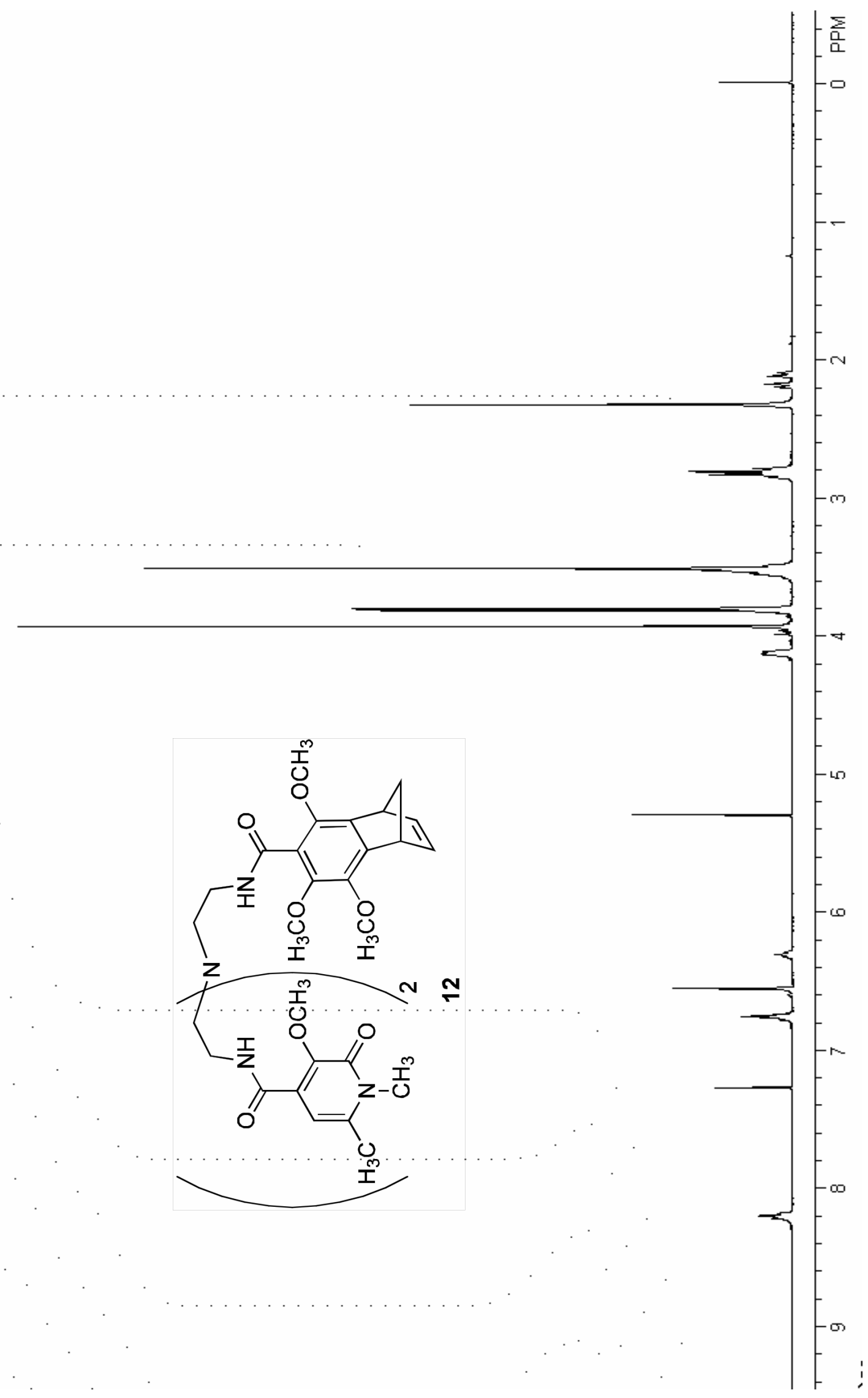




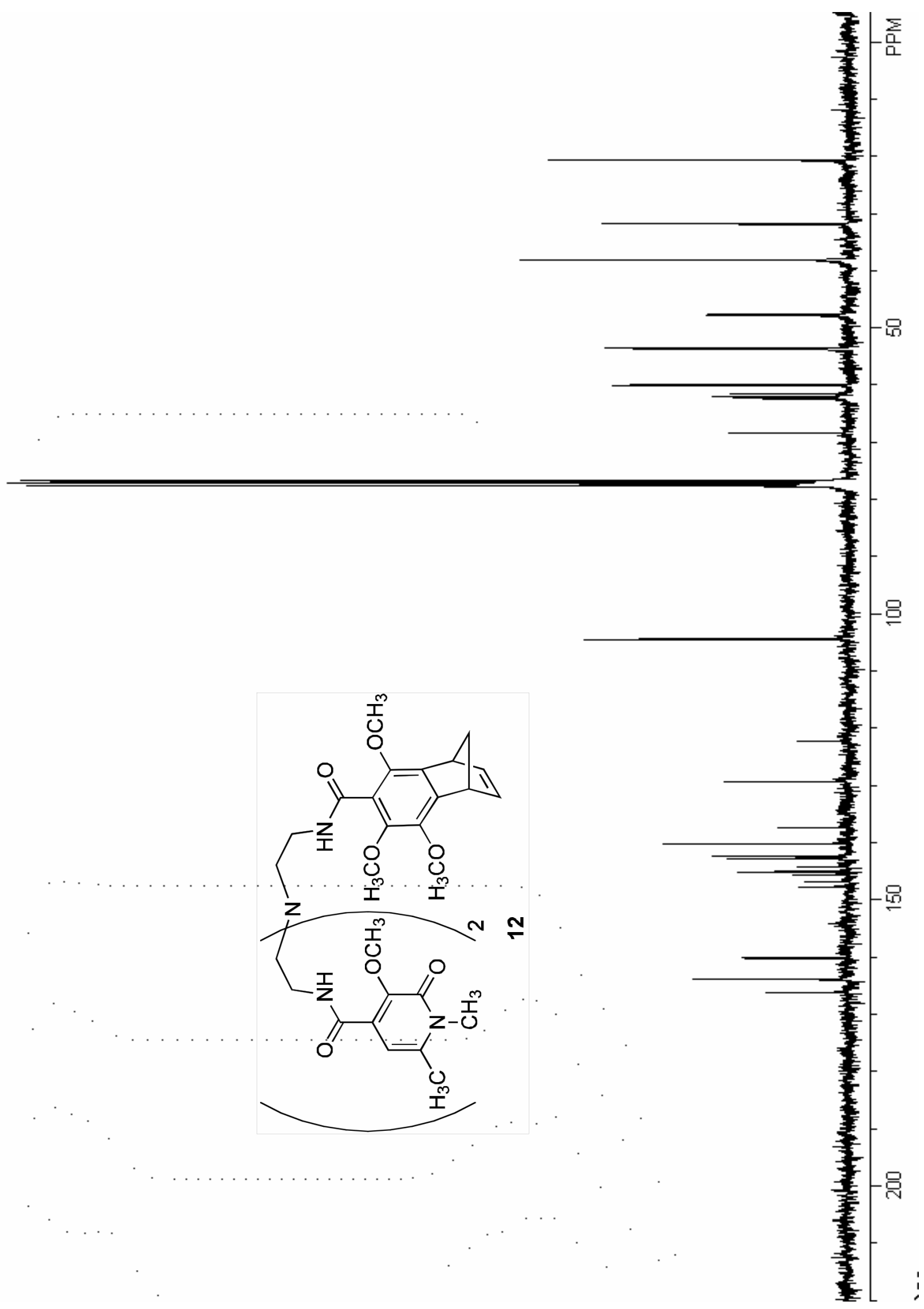




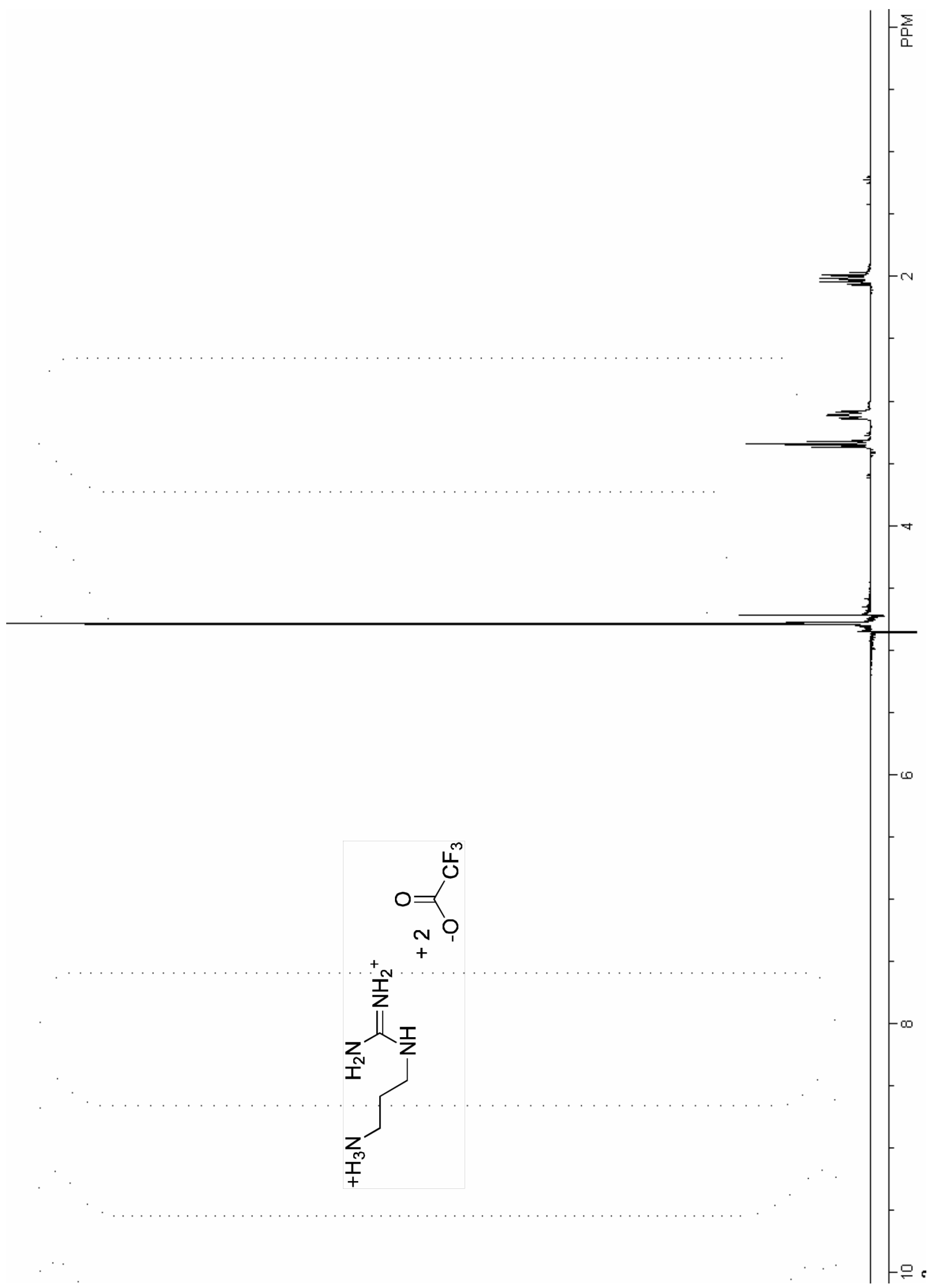




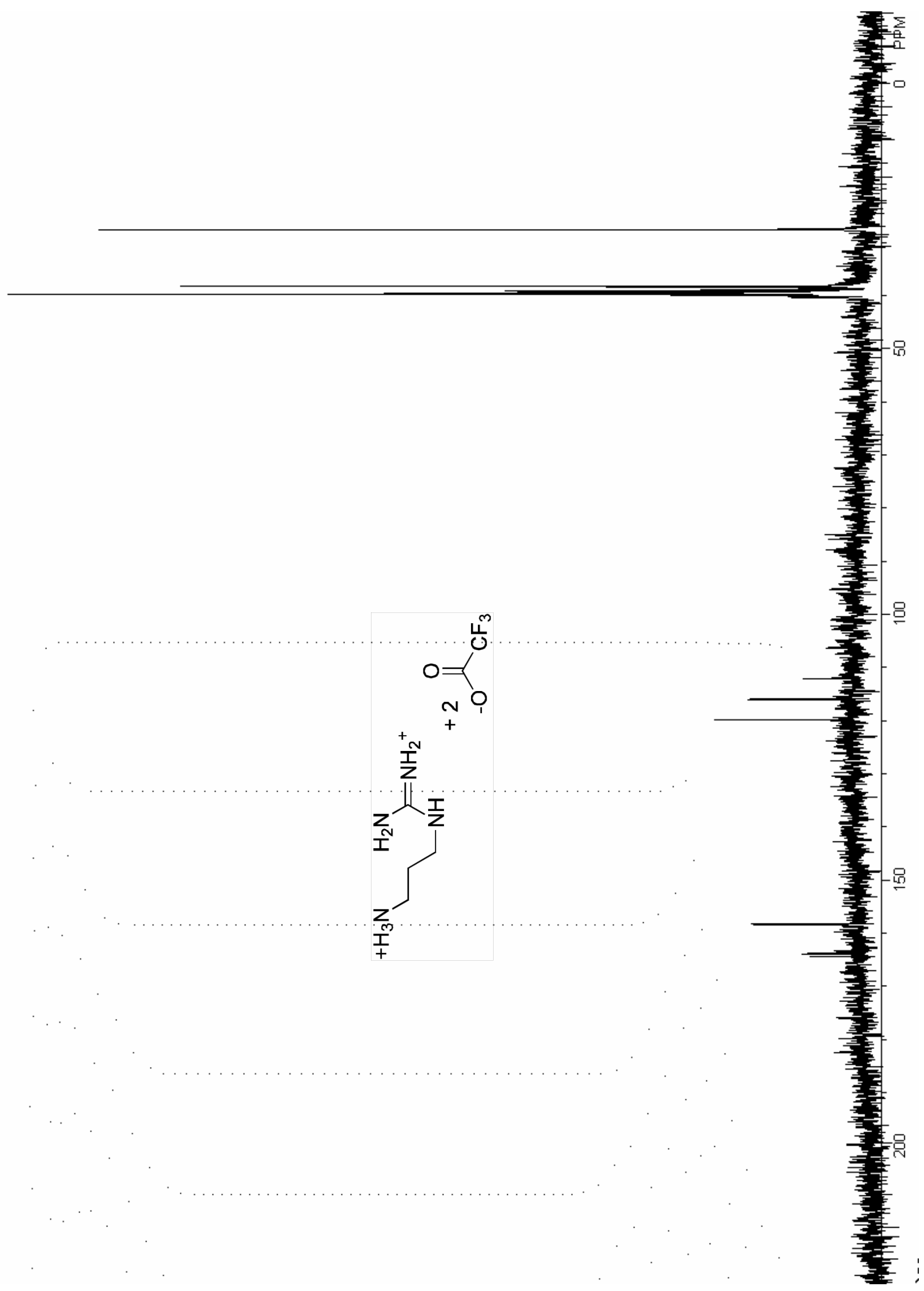




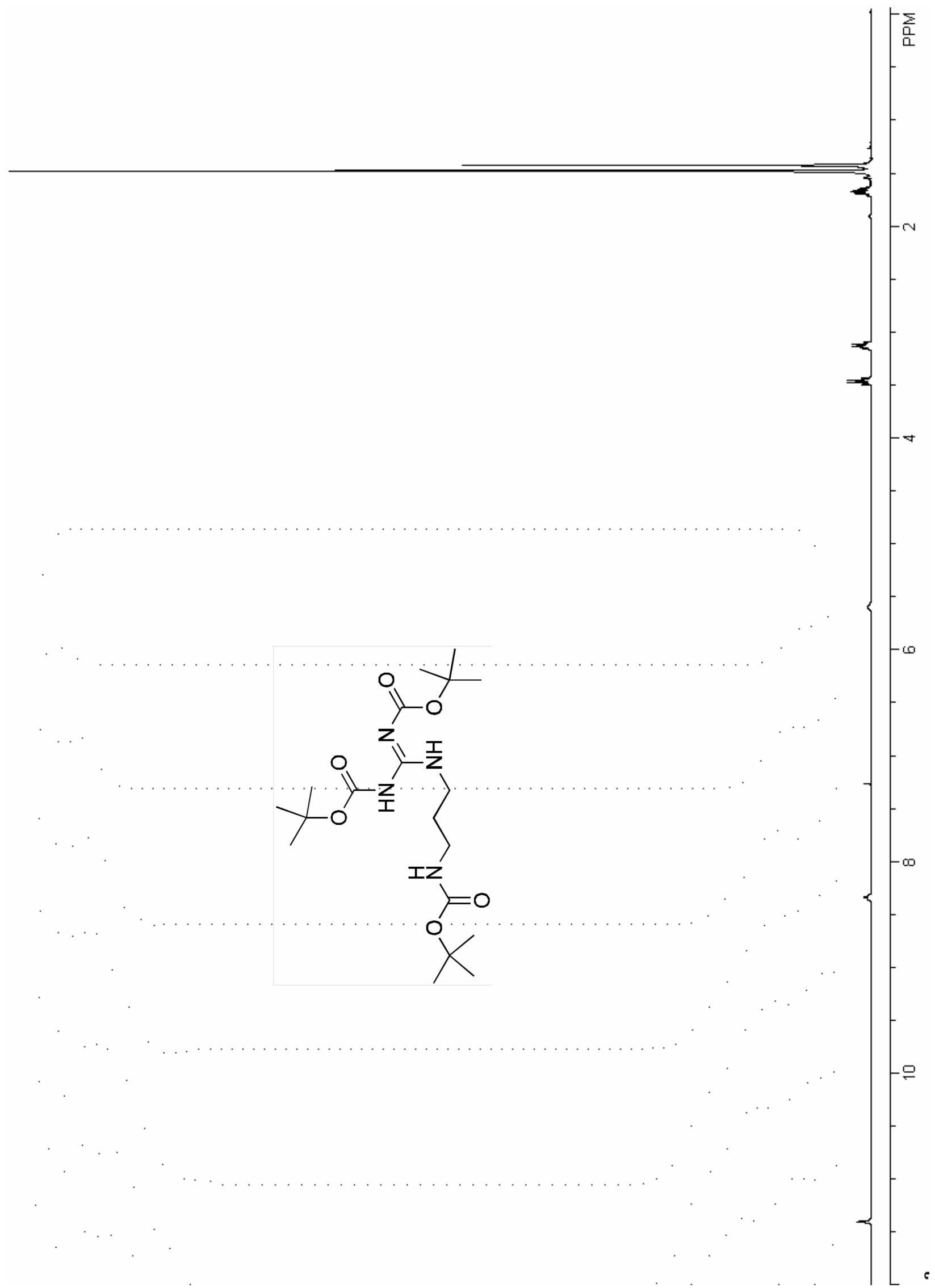






